This item was submitted to Loughborough's Research Repository by the author.

Items in Figshare are protected by copyright, with all rights reserved, unless otherwise indicated.

\title{
A game theoretic distributed algorithm for FeICIC optimization in LTE-A HetNets
}

PLEASE CITE THE PUBLISHED VERSION

https://doi.org/10.1109/TNET.2017.2748567

PUBLISHER

IEEE

VERSION

AM (Accepted Manuscript)

\section{PUBLISHER STATEMENT}

(c) 2017 IEEE. Personal use of this material is permitted. Permission from IEEE must be obtained for all other uses, in any current or future media, including reprinting/republishing this material for advertising or promotional purposes, creating new collective works, for resale or redistribution to servers or lists, or reuse of any copyrighted component of this work in other works

\section{LICENCE}

\section{All Rights Reserved}

\section{REPOSITORY RECORD}

Liu, Ye, Chung Shue Chen, Chi Wan Sung, and Chandramani Singh. 2019. "A Game Theoretic Distributed Algorithm for Feicic Optimization in LTE-A Hetnets”. figshare. https://hdl.handle.net/2134/27331. 


\title{
A Game Theoretic Distributed Algorithm for FeICIC Optimization in LTE-A HetNets
}

\author{
Ye Liu, Member, IEEE, Chung Shue Chen, Senior Member, IEEE, Chi Wan Sung, Senior Member, IEEE, and \\ Chandramani Singh, Member, IEEE
}

\begin{abstract}
In order to obtain good network performance in Long Term Evolution-Advanced (LTE-A) heterogeneous networks (HetNets), enhanced inter-cell interference coordination (eICIC) and further enhanced inter-cell interference coordination (FeICIC) have been proposed by LTE standardization bodies to address the entangled inter-cell interference and the user association problems. We propose distributed algorithms based on the exact potential game framework for both eICIC and FeICIC optimizations. We demonstrate via simulations a $64 \%$ gain on energy efficiency (EE) achieved by eICIC and another $17 \%$ gain on EE achieved by FeICIC. We also show that FeICIC can bring other significant gains in terms of cell-edge throughput, spectral efficiency (SE) and fairness among user throughputs. Moreover, we propose a downlink scheduler based on a cakecutting algorithm that can further improve the performance of the optimization algorithms compared to conventional schedulers.
\end{abstract}

Index Terms-LTE/LTE-A, heterogeneous networks, resource allocation, distributed optimization, potential game.

\section{INTRODUCTION}

According to an estimate of the growth of mobile data volume [1], more capacity must be added to the current cellular networks. Cell densification, due to its ability of reusing spectrum geographically and its property of preserving signal-tointerference-plus-noise ratio (SINR) [2], serves as a promising candidate solution to meet the demand of mobile users [3]. Contrary to the traditional cell densification where more highpower base stations (BSs) are added, it is more practical to add low-power BSs due to the high cost of installing macro BSs and the shortage of available sites suitable for macro BSs [4], which gives rise of the development of heterogeneous networks (HetNets).

The emergence of HetNets gives rise to two challenging network management problems. First, because pico BSs transmit

Y. Liu is with the Wolfson School of Mechanical, Manufacturing and Electrical Engineering, Loughborough University, Leicestershire, United Kingdom, LE113TU (email: y.liu6@lboro.ac.uk).

C. S. Chen is with the Mathematics of Dynamic \& Complex Networks department in Nokia Bell Labs, 91620 Nozay, France (e-mail: chung_shue.chen@nokia.com).

C. W. Sung is with the Department of Electronic Engineering, College of Science and Engineering, City University of Hong Kong, Kowloon, Hong Kong (e-mail: albert.sung@cityu.edu.hk).

C. Singh is with the Department of Electronic Systems Engineering, Indian Institute of Science (e-mail:chandra@iisc.ac.in).

Part of the work was done when Y. Liu was with Nokia Bell Labs, Centre de Villarceaux, 91620 Nozay, France.

This work has been partially supported by ANR project IDEFIX under grant number ANR-13-INFR-0006, a grant from the Research Grants Council of the Hong Kong Special Administrative Region, China, under Project CityU 121713, and by the Engineering and Physical Science Research Council of the UK, EPSRC, under the grant EP/M015475. at low power levels compared to macro BSs, mobile users who are physically located near pico BSs may be attracted to macro BSs, which can create underutilized pico BSs and overcrowded macro BSs. Therefore, in order to fully utilize the available resources in BSs with different transmission power, careful treatment is needed when performing user association. Second, the surrounding macro BSs of a pico BS can generate large interference to a user associated to the pico BS, and such inter-cell interference must be well-managed in order to prevent pico BSs' users from suffering very low downlink throughputs. To solve these issues, enhanced intercell interference coordination (eICIC) has been proposed in Release-10 of the 3GPP LTE standards, where

1) Cell selection bias (CSB) is used to offset the received signal power from BSs to a user so that a user is not necessarily associated with the BS that provides the strongest received power, and

2) Almost blank subframe (ABS) can be configured in macro BSs so that the macro BSs cease data transmissions in certain time slots, which reduces interference to pico BSs.

The use of ABSs can help reduce the interference from macro BSs to pico BSs. However, the restriction that macro BSs must mute their data transmissions entirely in ABSs may result in the inefficient use of the increasingly scarce resources. In Release-11, further enhanced inter-cell interference coordination (FeICIC) has been proposed, where instead of offering ABSs, macro BSs allocate reduced power almost blank subframes (RP-ABSs) to serve their users at reduced power levels.

Clearly, the configurations of CSB values and ABS patterns in eICIC optimization are coupled, because the amount of ABSs depends on the load on pico BSs which depends on the CSB values. To achieve the maximum possible performance gain using eICIC, joint optimization in ABS patterns and CSB values is required. Similarly, we must jointly consider RP-ABS patterns and CSB values when doing FeICIC optimization. While eICIC optimization algorithms have been studied in [5]-[15], little attention is paid on the algorithm that performs FeICIC optimization.

In this paper, we propose an exact potential game framework that is suitable for performing both eICIC and FeICIC optimizations. Specifically, we make the following contributions:

1) A distributed optimization framework: Based on the exact potential game framework, we propose a scalable distributed algorithm that can either jointly optimize 
ABS and CSB patterns or jointly optimize RP-ABS and CSB patterns. The game theoretic framework can adapt itself to various system optimization targets, such as proportional fairness $(\mathrm{PF})$ and sum rate maximization.

2) Performance evaluation: We evaluate the performance gain due to FeICIC and eICIC optimizations. Simulation results show that, compared to the case when no optimization is performed, FeICIC can nearly double the energy efficiency (EE) while eICIC provides about a $64 \%$ improvement on EE. Also, FeICIC provides higher fairness in the throughputs of the users and better celledge throughputs compared to eICIC.

3) A better downlink scheduler: We propose a downlink scheduler based on a cake-cutting algorithm. Simulation results show that the proposed scheduler can further improve the EE and spectral efficiency (SE) by $10 \%$ compared to conventional schedulers, can provide better fairness in SE, and is about 20 times faster than conventional convex algorithms in terms of simulation run time.

\section{A. Related work}

A number of eICIC optimization algorithms have been proposed in the literature. Tall et al.'s algorithm in [5] decouples the ABS optimization and CSB optimization, where the ABS patterns are simplified as fractional numbers. A centralized algorithm is proposed by Deb et al. in [6], where ABS and CSB patterns are jointly optimized and the surrounding macro BSs of a pico BS must offer ABSs on the same subframes. In [7], a distributed algorithm is proposed by Pang et al. where the number of ABSs is determined without considering CSB. Thakur et al. considered the problem of CSB optimization and power control in [8]. Bedekar and Agrawal, in [9], simplify the joint $\mathrm{ABS}$ and $\mathrm{CSB}$ optimization problem so that the optimization of ABS ratios and user attachment are solved separately. Simsek et al. propose a learning algorithm that optimizes CSB patterns in frequency domain in [10] and further extend the idea to optimizing CSB patterns in both time and frequency domain in [11]. Liu et al., in [12], propose to optimize the probability that a macro BS offers almost blank resource blocks on both time and frequency dimensions. Potential game based solutions for distributed eICIC optimization are considered in [13]-[15].

The benefit of FeICIC against eICIC has been analyzed in [16] using stochastic geometric approach, where the expressions for SE and cell-edge throughputs have been derived as a function of the power reduction factor on the RP-ABSs. However, the power reduction factor on all RP-ABSs are assumed to be the same in [16]. An optimization algorithm that can dynamically adjust the transmission power on each RP-ABS has not been considered to our best knowledge.

In this work, we address the FeICIC optimization problem based on exact potential game models. We adapt the game theoretic frameworks in [14], [15] such that power control on each time-frequency slot, i.e., physical resource block (PRB), are included during the optimization process. Also, we rigorously discuss the necessary assumptions which are needed for the validity of the exact potential game formulations and evaluate the effect of such assumptions. Moreover, we evaluate the performance of a downlink scheduler based on a cake-cutting algorithm and compare it against conventional schedulers.

\section{B. Organization and notation}

The rest of the paper is organized as follows. Section II gives the system model of the LTE-A HetNets. Section III formulates the eICIC and FeICIC optimization problems. Section IV develops the exact potential game framework that is suitable for eICIC and FeICIC optimizations. Section V describes the strategy sets and the better response dynamics of the games for eICIC and FeICIC optimization. Section VI introduces the cake-cutting downlink scheduler and other benchmark schedulers. Section VII presents the numerical studies. Finally, Section VIII concludes the paper.

Unless otherwise specified, we use small letters such as $a$ to denote scalars, bold small letters such as a to denote vectors, calligraphy letters such as $\mathcal{A}$ to denote sets. Also, $|\mathcal{A}|$ returns the number of elements in set $\mathcal{A}$ and $\emptyset$ denotes the empty set. $\mathcal{A} \backslash \mathcal{B}$ gives the elements in set $\mathcal{A}$ that are not in set $\mathcal{B}$.

\section{SYSTEM MODEL}

Consider a randomly generated HetNet as shown in Fig. 1 which consists of macro BSs and pico BSs, where the squares represent macro BSs and the triangles represent pico BSs. Denote $\mathcal{M}$ and $\mathcal{P}$ as the set of all macro BSs and the set of all pico BSs, respectively. Also, denote $\mathcal{M}_{\mathrm{c}}$ and $\mathcal{P}_{\mathrm{c}}$ as the macro BSs in the center cluster of the HetNet and pico BSs in the center cluster of the HetNet, respectively, where the center cluster is surrounded by bolded borders in Fig. 1 . Six clusters which are identical to the center clusters are placed around the center cluster. We make such distinction between the center cluster and other clusters because we only care about the optimization of the BSs in the center cluster, and the surrounding clusters are generated only to realize the interference as encountered in practice. We assume that there is only one macro BS located at the center of each hexagon, and each hexagon has the same number of pico BSs, e.g., one pico BS per hexagon in Fig. 1.

Let $\mathcal{N}(i, n)$ be BS $i$ 's neighboring BSs that are located in the $n$-th layer of hexagons w.r.t. ${ }^{1}$ the hexagon in which BS $i$ is located, where $i \in \mathcal{M} \cup \mathcal{P}$. The 0 -th layer of hexagons w.r.t. the hexagon $\xi$ is $\xi$ itself, and the $n$-th layer of hexagons w.r.t. $\xi$ are the hexagons

1) that are adjacent to the $(n-1)$-th layer of hexagons of $\xi$, and

2) that are further away from $\xi$ than the hexagons of the $(n-1)$-th layer.

For example, in Fig. $1, \mathcal{N}(1,0)$ gives $\{101\}$, $\mathcal{N}(101,0)$ gives $\{1\}$, both $\mathcal{N}(1,1)$ and $\mathcal{N}(101,1)$ give $\{2,3,4,5,6,7,102,103,104,105,106,107\}$, and both $\mathcal{N}(1,2)$ and $\mathcal{N}(101,2)$ give the set of BSs in the center cluster except the BSs in $\{1,101\} \cup \mathcal{N}(1,1)$. The definition

\footnotetext{
${ }^{1}$ w.r.t. stands for with respect to.
} 


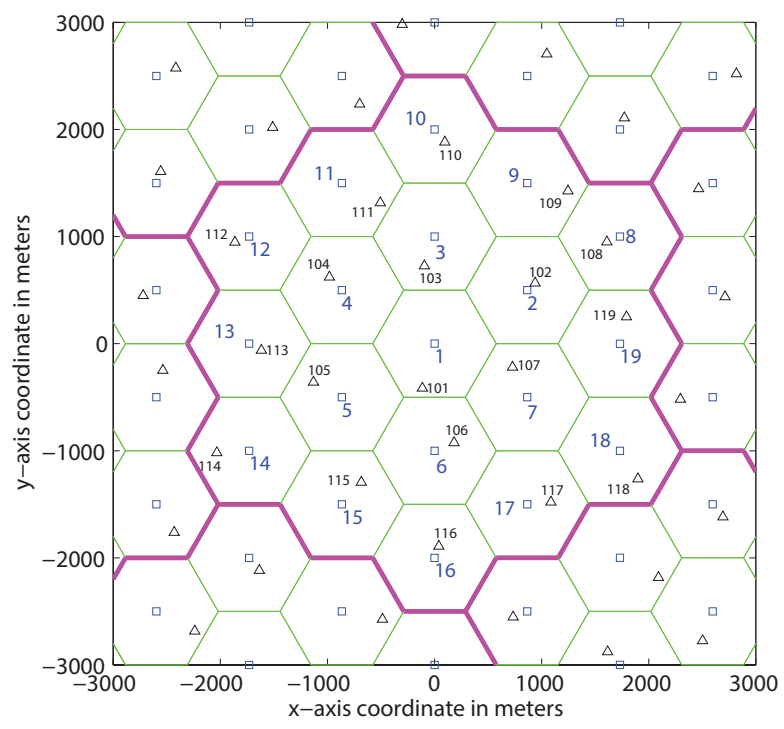

Fig. 1: An example of a hexagonal HetNet layout. The squares represent the macro BSs and the triangles represent the pico BSs. Users are not displayed for the sake of clarity.

of $\mathcal{N}(i, n)$ can also be easily extended to the case where $i$ represents a set of BSs located in the same hexagon.

Let $\mathcal{U}$ be the set of all users in the system. Denote $m_{u}$ as the macro BS that is located in the same hexagon as user $u$. We assume that only the BSs in the same hexagon or in the adjacent hexagons can serve a user. In other words, the set of candidate BSs that can serve user $u$ is given as:

$$
\mathcal{O}_{u} \triangleq\left\{m_{u}\right\} \cup \mathcal{N}\left(m_{u}, 0\right) \cup \mathcal{N}\left(m_{u}, 1\right) .
$$

Define vector $\gamma_{\mathcal{O}_{u}}$ as the CSB values of all BSs in $\mathcal{O}_{u}$ and let $\gamma_{\mathcal{O}_{u}}(i)$ gives the CSB value of BS $i$, where $i \in \mathcal{O}_{u}$. The set $\mathcal{C}$ contains all possible values that $\gamma_{\mathcal{O}_{u}}(i)$ can take. Let $P_{i, u}^{\mathrm{Rx}}$ be the reference signal received power (RSRP) of user $u$ from BS $i$ when the BS is transmitting at its full power. The exact value of $P_{i, u}^{\mathrm{Rx}}$ depends on the distance between BS $i$ and user $u$ and the loss due to shadow fading. The effect of fast fading is assumed to be averaged out for $P_{i, u}^{\mathrm{Rx}}$. The following equation gives the BS that serves user $u$ :

$$
g\left(u, \boldsymbol{\gamma}_{\mathcal{O}_{u}}\right) \triangleq \arg \max _{i \in \mathcal{O}_{u}}\left(P_{i, u}^{\mathrm{Rx}}+\boldsymbol{\gamma}_{\mathcal{O}_{u}}(i)\right)
$$

Let $\mathcal{U}_{\mathcal{B}}$ be the set of users who are associated with BSs in the set $\mathcal{B}$, i.e.,

$$
\mathcal{U}_{\mathcal{B}} \triangleq\left\{u \mid g\left(u, \boldsymbol{\gamma}_{\mathcal{O}_{u}}\right) \in \mathcal{B}\right\} .
$$

Clearly, $\mathcal{U}_{\mathcal{B}}$ is a function of the CSB values of the BSs in $\mathcal{B}$ and their nearby BSs. Let $\gamma$ denote the vector which specifies all BS's CSB values.

Suppose each BS has $N_{T}$ subframes in the time domain and $N_{F}$ resource blocks (RBs) in the frequency domain. All subframes have the same duration and all RBs are identical in terms of bandwidth. A PRB is formed by a pair of subframe and RB, and we denote $N_{B}:=N_{T} \cdot N_{F}$ as the total number of PRBs available at each BS. It is assumed that all subframes and RBs of all BSs are synchronized.
Let the length $N_{T}$ vector $\boldsymbol{\alpha}_{m}$ specify the ABS pattern of macro BS $m$, where all the entries in $\boldsymbol{\alpha}_{m}$ are binary. Let $\mathcal{A}$ contain all possible ABS patterns that a macro BS can adopt, where each element in $\mathcal{A}$ consists of a binary vector of length $N_{T}$. Also, let $\hat{\mathcal{A}}$ be a subset of $\left\{1,2, \ldots, N_{T}\right\}$ which contains the indices of subframes which can be an ABS as indicated in any element in $\mathcal{A}$. For example, suppose $\mathcal{A}=\{(0,1,1,1),(0,1,0,1),(0,0,0,1)\}$, then $\hat{\mathcal{A}}=\{1,2,3\}$ because subframes 1,2 , and 3 are possible ABSs.

Let $\tau(b)$ be the subframe index of PRB $b$. Moreover, let $\hat{\boldsymbol{\alpha}}_{m}$ be a vector of length $N_{T} \times N_{F}$ whose elements specify the power allocation of macro BS $m$ on each PRB, where $\hat{\boldsymbol{\alpha}}_{m}(b)$ is a real number between 0 and 1 for $\tau(b) \in \hat{\mathcal{A}}$ and $\hat{\boldsymbol{\alpha}}_{m}(b)$ is fixed to be one for $\tau(b) \in\left\{1,2, \ldots, N_{T}\right\} \backslash \hat{\mathcal{A}}$. The vector $\hat{\boldsymbol{\alpha}}_{m}$ then defines the RP-ABS pattern of macro BS $m$. Note that although it is not necessary to assume that a macro BS offers RP-ABS only in the subframes specified by $\hat{\mathcal{A}}$, the definition of $\hat{\boldsymbol{\alpha}}_{m}$ aims at offering a fair comparison between FeICIC optimization and eICIC optimization.

In this paper, we assume that only the macro BSs would offer ABSs/RP-ABSs while the pico BSs always transmit on all subframes. Such an assumption is reasonable because

1) The macro BSs have much more transmission power than the pico BSs. Consequently, the macro BSs are the main source of interference in the network.

2) The complexity of the resulting eICIC/FeICIC optimization is reduced compared to the case where all stations offer ABSs/RP-ABSs.

Also, we assume that only the pico BSs may set their CSB values to some positive numbers while the macro BSs fix their CSB values to zeros. This is because, in general, it is the coverage range of a pico BS which needs to be extended in order to better utilize the resources from the pico BS.

Given the above definitions, the signal-to-noise-plusinterference ratio (SINR) of user $u$ on PRB $b$ when associated with macro BS $m$ can be calculated as:

$$
\operatorname{SINR}_{u, b}^{m}=\left\{\begin{array}{l}
\frac{h_{u, b}^{m} P_{m, u}^{\mathrm{Rx}} \cdot \boldsymbol{\alpha}_{m}(\tau(b))}{P_{\mathcal{I}_{m}, u, b}^{\mathrm{IF}}+N_{0} W}, \text { BS } m \text { offers ABS, (2a) } \\
\frac{h_{u, b}^{m} P_{m, u}^{\mathrm{Rx}} \cdot \hat{\boldsymbol{\alpha}}_{m}(b)}{P_{\mathcal{I}_{m}, u, b}^{\mathrm{IF}}+N_{0} W}, \text { BS } m \text { offers RP-ABS,(2b) }
\end{array}\right.
$$

where $h_{u, b}^{m}$ gives the fast fading gain on PRB $b$ from macro BS $m$ to user $u, \tau(b)$ returns the subframe index of PRB $b, \mathcal{I}_{m}$ denotes the set of BSs whose transmission will interfere the users located in the same hexagon as macro BS $m, P_{\mathcal{I}_{m}, u, b}^{\mathrm{IF}}$ is the sum of interference at user $u$ received from BSs in $\mathcal{I}_{m}$ at PRB $b, N_{0}$ denotes the additive white Gaussian noise (AWGN) spectral density and $W$ is the bandwidth of a PRB. Similarly, the SINR of user $u$ on PRB $b$ when associated with pico BS $p$ is given by:

$$
\operatorname{SINR}_{u, b}^{p}=\frac{h_{u, b}^{p} P_{p, u}^{\mathrm{Rx}}}{P_{\mathcal{I}_{p}, u, b}^{\mathrm{IF}}+N_{0} W},
$$

where a pico BS does not offer ABS/RP-ABS as discussed before. Let $r_{u, b}$ be the achieved rate of user $u$ at PRB $b$, where $b \in\left[1, N_{B}\right]$. It is assumed that the serving BS knows 
the achieved rate of user $u$ at $\operatorname{PRB} b$, and the achieved rate is calculated by Shannon's capacity formula, i.e.,

$$
r_{u, b}= \begin{cases}W \cdot \log _{2}\left(1+\operatorname{SINR}_{u, b}^{m}\right), & g\left(u, \gamma_{\mathcal{O}_{u}}\right)=m \in \mathcal{M}, \\ W \cdot \log _{2}\left(1+\operatorname{SINR}_{u, b}^{p}\right), & g\left(u, \gamma_{\mathcal{O}_{u}}\right)=p \in \mathcal{P} .\end{cases}
$$

Table I summarizes the notation used in this paper.

\section{PROBLEM Formulation}

Let $x_{u, b}$ be a binary variable indicating whether PRB $b$ is allocated to user $u$ by its serving $\mathrm{BS}$, where $x_{u, b}=1$ means that PRB $b$ is allocated to user $u$ and $x_{u, b}=0$ means otherwise. To discriminate the importance of different users, positive weighting factors are applied, where we denote $w_{u}$ as the weighting factor for user $u$.

We formulate the eICIC optimization problem as follows

\section{MaxPfUtility-I}

$$
\begin{aligned}
& \operatorname{maximize} \sum_{i \in \mathcal{M} \cup \mathcal{P}} \sum_{u \in \mathcal{U}_{i}} w_{u} \cdot \ln \sum_{b=1}^{N_{B}}\left(x_{u, b} \cdot r_{u, b}\right) \text {, } \\
& \text { subject to } \sum_{u \in \mathcal{U}_{m}} x_{u, b}=\boldsymbol{\alpha}_{m}(\tau(b)) \text {, } \\
& \forall m \in \mathcal{M}_{c}, b \in\left[1, N_{B}\right], \boldsymbol{\alpha}_{m} \in \mathcal{A}, \\
& \sum_{u \in \mathcal{U}_{p}} x_{u, b}=1, \forall p \in \mathcal{P}_{c}, b \in\left[1, N_{B}\right] \text {, } \\
& x_{u, b} \in\{0,1\}, \forall u \in \mathcal{U}, b \in\left[1, N_{B}\right] \text {, } \\
& \gamma(i) \in \mathcal{C}, \forall i \in \mathcal{P}_{c},
\end{aligned}
$$

where (4b) specifies that a macro BS can adopt one of the ABS patterns in $\mathcal{A}$ and only non-ABS PRBs can be assigned to the users such that at most one user can occupy a PRB, (4c) states that all PRBs from pico BSs can be allocated to the users and at most one user can occupy a PRB, and (4e) means that a pico BS can adopt one of the CSB values specified in $\mathcal{C}$.

For the FeICIC optimization in which macro BSs may offer RP-ABSs, we aim at solving the following problem

\section{MaXPFUTILITY-II}

$$
\begin{aligned}
\operatorname{maximize} & \sum_{i \in \mathcal{M} \cup \mathcal{P}} \sum_{u \in \mathcal{U}_{i}} w_{u} \cdot \ln \sum_{b=1}^{N_{B}}\left(x_{u, b} \cdot r_{u, b}\right), \\
\text { subject to } & \hat{\boldsymbol{\alpha}}_{m}(b) \in[0,1], \tau(b) \in \hat{\mathcal{A}}, \forall m \in \mathcal{M}_{c}, \\
& \hat{\boldsymbol{\alpha}}_{m}(b)=1, \tau(b) \in\left\{1,2, \ldots, N_{T}\right\} \backslash \hat{\mathcal{A}}, \forall m \in \mathcal{M}_{c}, \\
& \sum_{u \in \mathcal{U}_{i}} x_{u, b}=1, \forall i \in \mathcal{M}_{c} \cup \mathcal{P}_{c}, b \in\left[1, N_{B}\right],
\end{aligned}
$$

(4d) and (4e),

where (5b) means that power allocation is optimized on PRBs whose subframe indices are in $\hat{\mathcal{A}}$ and (5c) means that no power optimization is performed on PRBs whose subframe indices are not in $\hat{\mathcal{A}}$. Because there is no restriction on a macro BS that it must completely mute its transmission on a subframe

\begin{tabular}{|c|c|}
\hline Notation & Description \\
\hline $\boldsymbol{\alpha}_{m}$ & ABS pattern of macro BS $m$ \\
\hline$\hat{\boldsymbol{\alpha}}_{m}$ & RP-ABS pattern of macro BS $m$ \\
\hline$\gamma$ & Vector specifying CSB values of all BSs \\
\hline$\gamma_{\mathcal{O}_{u}}$ & Vector specifying CSB values of BSs in $\mathcal{O}_{u}$ \\
\hline$g\left(u, \gamma_{\mathcal{O}_{u}}\right)$ & The BS that user $u$ is associated with \\
\hline$m_{i}$ & $\begin{array}{l}\text { The macro BS located in the same hexagon as an object } \\
\text { with index } i \text {, where the object can be a user or a pico BS }\end{array}$ \\
\hline$r_{u, b}$ & Achieved rate of user $u$ at PRB $b$ \\
\hline$\tau(b)$ & The subframe index of PRB $b$ \\
\hline$w_{u}$ & Weighting factor on the achieved rate of UE $u$ \\
\hline$x_{u, b}$ & $\begin{array}{c}\text { Indicator of whether user } u \text { occupies PRB } b \text { of the } \\
\text { serving cell }\end{array}$ \\
\hline$N_{0}$ & Noise power spectral density \\
\hline$N_{B}$ & Number of PRBs \\
\hline$N_{F}$ & Number of RBs (in frequency domain) \\
\hline$N_{T}$ & Number of subframes (in time domain) \\
\hline$V_{i}$ & The payoff function of player $i$ \\
\hline $\bar{W}$ & Bandwidth per RB \\
\hline $\mathcal{A}$ & $\begin{array}{l}\text { Set of vectors from which macro BSs can } \\
\text { choose their ABS patterns }\end{array}$ \\
\hline$\hat{\mathcal{A}}$ & Set containing indices of subframes which can be ABSs \\
\hline $\mathcal{C}$ & Set of CSB values from which a pico BS can choose from \\
\hline $\mathcal{I}_{m}$ & $\begin{array}{l}\text { Set of BSs whose transmissions interfere the users } \\
\text { located in the same hexagon as BS } m\end{array}$ \\
\hline $\mathcal{L}$ & The set of players in the potential game model \\
\hline $\mathcal{M}$ & Set of all macro BSs \\
\hline $\mathcal{M}_{\mathrm{c}}$ & Set of macro BSs in the center cluster \\
\hline $\mathcal{N}(i, n)$ & $\begin{array}{l}\text { The set of BS } i \text { 's neighboring BSs located in the } \\
n \text {-th layer of hexagons w.r.t. the hexagon that contains } i\end{array}$ \\
\hline $\mathcal{O}_{u}$ & Candidate BSs who can serve user $u$ \\
\hline $\mathcal{P}$ & Set of all pico BSs \\
\hline $\mathcal{P}_{\mathrm{c}}$ & Set of pico BSs in the center cluster \\
\hline $\mathcal{S}_{i}$ & The strategy set of player $i$ \\
\hline $\mathcal{U}$ & Set of all users in the system \\
\hline $\mathcal{U}_{i}$ & Set of users associated with BSs in set $i$ or with BS $i$ \\
\hline
\end{tabular}
in FeICIC optimization, every PRB from a macro BS can be allocated to at most one user as specified in $(5 \mathrm{~d})$.

The objective functions of both (4) and (5) are defined as the sum of logarithm of users' throughputs. Such an objective achieves the proportional fairness among the users' achievable rates, which strikes a good trade-off between aggregate network throughput and user fairness [17]. Also, different realizations of $\gamma$ will affect the elements in $\left\{\mathcal{U}_{i} \mid i \in \mathcal{P}_{c}\right\}$, which is how CSB optimization comes into the problems (4) and (5).
TABLE I: Summary of notation.

We now show the NP-hardness of (4) and (5).

Theorem 1. Both (4) and (5) are NP-hard.

Proof: Consider the case where no ABS/RP-ABS is applied in any macro BS and all pico BSs fix their CSB values to zeros, and assume there is only one element in $\mathcal{M}_{c} \cup \mathcal{P}_{c}$. We then obtain a special case for both (4) and (5) where the only problem left is to decide how to allocate the PRBs of a single BS. We denote this special case as:

\section{PRB-Allocation}

$$
\begin{aligned}
\operatorname{maximize} & \sum_{u \in \mathcal{U}_{i}} w_{u} \cdot \ln \sum_{b=1}^{N_{B}}\left(x_{u, b} \cdot r_{u, b}\right), \\
\text { subject to } & \sum_{u \in \mathcal{U}_{i}} x_{u, b}=1, b \in\left[1, N_{B}\right], \\
& x_{u, b} \in\{0,1\}, \forall u \in \mathcal{U}_{i}, b \in\left[1, N_{B}\right] .
\end{aligned}
$$

It is shown in [18] that (6) is NP-hard. Therefore, both (4) and (5) are NP-hard because a special case of the two problems is NP-hard.

In the next section, we propose a potential game based framework which can be applied to both (4) and (5) to solve the problems distributedly and heuristically.

\section{Exact Potential Game Formulation}

In this section, we frame the eICIC and FeICIC optimization problems as exact potential games. Our approach is motivated 
by the successful application of potential games to another scenario in [19] for BS power control and user association.

\section{A. Preliminary}

A finite game consists of a finite set of players, a finite set of strategies of each player, and the payoff functions of the players, where the payoff of a player is a function of the strategies played by all the players. A strategy profile gives the strategies adopted by all the players, and a Nash equilibrium is a strategy profile $\mathbf{s}^{*}$ such that no player can improve its payoff by playing a different strategy than the one specified in $\mathrm{s}^{*}$ while other players keep their strategies same.

A game is called an exact potential game if there exists an exact potential function such that change in the value of the exact potential function due to a change of a player's strategy is the same as the change of the player's payoff. In a finite exact potential game, a Nash equilibrium can be achieved if players take turns randomly and play their best responses or better responses [20], where, given that all other players fix their strategies,

1) A best response is a player strategy that maximizes the player's payoff function.

2) A better response is a player strategy that improves the payoff function of the player.

As demonstrated later, that being able to formulate the eICIC and FeICIC optimization problems as exact potential games will allow us to solve them distributively using simple algorithms based on best/better response dynamics.

In order to realize the process by which a macro BS adapts its ABS/RP-ABS pattern when a pico BS in the same hexagon optimizes its CSB value, it is convenient to define a player as a union of a macro BS and the pico BSs within the same hexagon. Let $\mathcal{L}$ be the set of players, where each element in $\mathcal{L}$ consists of a set that contains the macro BS and the pico BSs in a hexagon in the center cluster. We can then denote the game as $\Gamma \triangleq\left\langle\mathcal{L},\left\{\mathcal{S}_{i}: i \in \mathcal{L}\right\},\left\{V_{i},: i \in \mathcal{L}\right\}\right\rangle$, where $\mathcal{S}_{i}$ is the strategy set of player $i$ and $V_{i}$ is the payoff function of player $i$. Note that the game structure $\Gamma$ can be applied to both eICIC and FeICIC optimization problems because the two problems have the same players and the same objective functions. The only difference between the eICIC optimization and the FeICIC optimization is the power allocation constraint on the PRBs, and this difference can be captured by the definitions of the respective strategy sets. The details of the strategy sets and payoff functions will be discussed later.

When a player changes its strategy during the game for eICIC optimization, users that are associated with the BSs represented by the player and are associated with other nearby BSs would be affected. A similar situation applies to the game for FeICIC optimization. Consequently, to achieve a good system performance for both (4) and (5), the payoff function of a player should take users who are located in nearby hexagons into account, even if these users are not being served by the player. On the other hand, the transmission of a BS can, in theory, interfere users located very far away. To ensure accuracy, the payoff function of a player should then consider all users in the system. However, such a payoff function will introduce high complexity to the optimization process and at the same time deviate from the intention of designing a distributed algorithm. Some approximation on the interference is necessary for a low complexity distributed algorithm. It is therefore important to first identify the impact of changing ABS/RP-ABS and CSB patterns before defining a payoff function that leads to an exact potential game and facilitates low-complexity distributed designs.

In the following, we first discuss which neighboring BSs of player $i$ can be affected by changes in player $i$ 's CSB values $^{2}$. We then define the payoff function of players and identify an exact potential function based on some interference approximation. Details of the strategy sets, the algorithms that converge to a Nash equilibrium, and the downlink schedulers will be given in later sections.

\section{B. Neighboring sets of a player}

As mentioned in previous discussion, for scalability, we make an approximation that the interference range of a BS is limited only to some of its neighboring hexagons, because the interference power from a BS to a user is negligible if the user is located far away from the BS. We use $\mathcal{N}_{i}^{\mathrm{IF}}$ to specify the set of BSs whose hexagons are interfered by player $i$. More precisely, it means that a user is interfered by the transmission of the BSs represented by player $i$ if and only if he is located in the hexagon of a BS that belongs to $\mathcal{N}_{i}^{\mathrm{IF}}$.

Let $\mathcal{N}_{i}^{\text {Att }}$ contains the BSs whose user attachment patterns depend on the CSB values of the pico BSs represented by player $i$. Clearly, the actual serving BS of a user depends on the CSB values of the pico BSs represented by player $i$, if a BS represented by player $i$ is a candidate serving BS of that user. Moreover, because user $u$ can be attached to any BS in $\mathcal{O}_{u}$, the actual serving BS of user $u$ depends on the CSB values of all BSs in $\mathcal{O}_{u}$. Therefore,

$$
\mathcal{N}_{i}^{\text {Att }}=\bigcup_{\left\{\forall u \mid\{i\} \subset \mathcal{O}_{u}\right\}} \mathcal{O}_{u} .
$$

The next proposition shows which elements constitute $\mathcal{N}_{i}^{\text {Att }}$.

Proposition 1. $\mathcal{N}_{i}^{\text {Att }}=i \cup \mathcal{N}(i, 1) \cup \mathcal{N}(i, 2)$.

Proof: See Appendix A.

Define the utility of player $i$ as

$$
U_{i}(\mathbf{s}) \triangleq \sum_{u \in \mathcal{U}_{i}} w_{u} \cdot \ln \sum_{b=1}^{N_{B}}\left(x_{u, b} \cdot r_{u, b}\right)
$$

where $\mathbf{s}$ is the strategy vector that specifies the strategies played by all players. Let $\mathcal{N}_{i}$ contain player $i$ and player $i$ 's neighboring BSs whose downlink users' SINRs and/or whose user attachment patterns can be affected by changing the ABS/RP-ABS patterns and CSB values of player $i$. The next proposition shows the elements in $\mathcal{N}_{i}$ when $\mathcal{N}_{i}^{\mathrm{IF}}=i \cup \mathcal{N}(i, 1)$.

Proposition 2. Suppose $i \in \mathcal{L}$ and $\mathcal{N}_{i}^{I F}=i \cup \mathcal{N}(i, 1)$. Keeping $\mathbf{s}_{-i}$ unchanged, changes in $s_{i}$ may affect $U_{j}$ only if $j \in \mathcal{N}_{i}^{\text {Att }}$. In other words, $\mathcal{N}_{i}=\mathcal{N}_{i}^{\text {Att }}$.

Proof: See Appendix B.

\footnotetext{
${ }^{2}$ More accurately, by changes of the CSB values of the pico BSs represented by player $i$.
} 
The approximation on the interference range, i.e., the definition of $\mathcal{N}_{i}^{\mathrm{IF}}$, is crucial to the constitution of $\mathcal{N}_{i}$. This is demonstrated in the next proposition.

Proposition 3. Suppose $i \subset \mathcal{L}$ and $\mathcal{N}_{i}^{I F}=i \cup \mathcal{N}(i, 1) \cup\{j\}$, where $j \in \mathcal{N}(i, 2)$, then $\mathcal{N}_{i}^{A t t} \subset \mathcal{N}_{i}$.

Proof: See Appendix C.

\section{Exact potential game formulation}

The key to the exact potential game formulation lies in the appropriate definition of the payoff function. We first define some notations regarding the strategies of players before defining the payoff function. Then, we show there exists an exact potential function with respect to our payoff function.

Let $s_{i}$ be the strategy that player $i$ adopts, where $s_{i} \in \mathcal{S}_{i}$. Define

$$
\mathbf{s}_{-i} \triangleq\left(s_{1}, \ldots, s_{i-1}, s_{i+1}, \ldots, s_{\left|\mathcal{M}_{c}\right|}\right)
$$

to be the strategies of all players other than player $i$. Denote

$$
\left(\widetilde{s_{i}}, \mathbf{s}_{-i}\right) \triangleq\left(s_{1}, \ldots, s_{i-1}, \widetilde{s_{i}}, s_{i+1}, \ldots, s_{\left|\mathcal{M}_{c}\right|}\right)
$$

as the strategies of all players, where player $i$ selects strategy $\widetilde{s_{i}}$ and other players' strategies are specified as $\mathbf{s}_{-i}$. The payoff function of player $i$ is defined as

$$
V_{i}(\mathbf{s}) \triangleq \sum_{j \subset \mathcal{N}_{i}^{\text {Att }}} U_{j}(\mathbf{s}),
$$

and the aggregate utility of all the players is given as

$$
U(\mathbf{s})=\sum_{i \in \mathcal{L}} U_{i}(\mathbf{s}) .
$$

In the following theorem, we show that when $\mathcal{N}_{i}^{\mathrm{IF}}=i \cup$ $\mathcal{N}(i, 1), U(\mathbf{s})$ is an exact potential function.

Theorem 2. If $\mathcal{N}_{i}^{I F}=i \cup \mathcal{N}(i, 1)$, then $U(\cdot)$ is an exact potential function of the game $\Gamma$, such that $\Gamma$ is an exact potential game.

Proof: Suppose player $i$ changes its strategy, so that the strategies played by all players changes from $\mathbf{s}$ to $\left(\widetilde{s_{i}}, \mathbf{s}_{-i}\right)$. The change in $U(\cdot)$ due to this unilateral change of player $i$ 's strategy is:

$$
\begin{aligned}
& U\left(\widetilde{s}_{i}, \mathbf{s}_{-i}\right)-U(\mathbf{s}) \\
= & \sum_{j \subset \mathcal{L}}\left(U_{j}\left(\widetilde{s_{i}}, \mathbf{s}_{-i}\right)-U_{j}(\mathbf{s})\right) \\
= & \sum_{j \subset \mathcal{N}_{i}^{\text {Att }}}\left(U_{j}\left(\widetilde{s_{i}}, \mathbf{s}_{-i}\right)-U_{j}(\mathbf{s})\right)+\sum_{j \subset \mathcal{L} \backslash \mathcal{N}_{i}^{\text {Att }}}\left(U_{j}\left(\widetilde{s_{i}}, \mathbf{s}_{-i}\right)-U_{j}(\mathbf{s})\right) \\
= & \sum_{j \subset \mathcal{N}_{i}}\left(U_{j}\left(\widetilde{s_{i}}, \mathbf{s}_{-i}\right)-U_{j}(\mathbf{s})\right)+\sum_{j \subset \mathcal{L} \backslash \mathcal{N}_{i}}\left(U_{j}\left(\widetilde{s_{i}}, \mathbf{s}_{-i}\right)-U_{j}(\mathbf{s})\right) \\
= & \sum_{j \subset \mathcal{N}_{i}}\left(U_{j}\left(\widetilde{s_{i}}, \mathbf{s}_{-i}\right)-U_{j}(\mathbf{s})\right) \\
= & \sum_{j \subset \mathcal{N}_{i}^{\text {Att }}}\left(U_{j}\left(\widetilde{s_{i}}, \mathbf{s}_{-i}\right)-U_{j}(\mathbf{s})\right) \\
= & V_{i}\left(\left(\widetilde{s_{i}}, \mathbf{s}_{-i}\right)\right)-V_{i}(\mathbf{s})
\end{aligned}
$$

where (11a) and (11c) follow from Proposition 2, (11b) follows from the definition of $\mathcal{N}_{i}$, and (11d) follows from the definition of the payoff function. Equation (11d) indicates that the change of $U(\cdot)$ due to the change of a player's strategy is exactly the same the change of the payoff function of that player. This proves that $U(\cdot)$ is an exact potential function of the game $\Gamma$. Consequently, $\Gamma$ is an exact potential game because it admits an exact potential function.

Note that the above potential game framework can also be used to optimize utility functions other than proportional fairness. For example, in case the objective function in (4) and (5) is the sum of all users' rates, then the same potential game framework can still be used except that now the utility function of player $i$ should be $\sum_{u \in \mathcal{U}_{i}} w_{u} \cdot \sum_{b=1}^{N_{B}}\left(x_{u, b} \cdot r_{u, b}\right)$.

\section{Strategy Sets and Optimization Algorithms}

In this section, we define the strategy sets of the players for the eICIC and FeICIC optimizations based on exact potential game formulations. We also provide the algorithms that solve the exact potential games for eICIC and FeICIC optimizations.

\section{A. The strategy sets and the algorithm for eICIC}

By definition, $r_{u, b}$ is a function of the ABS patterns of the macro BSs in $\mathcal{N}_{i}^{\mathrm{IF}}$ and the CSB values of the pico BSs in $\mathcal{N}_{i}^{\text {Att }}$, where $i \subset \mathcal{L}$. Moreover, $x_{u, b}$ is a function of the downlink scheduler of the serving BS of user $u$. Therefore, the strategy of a player should specify the ABS pattern of the macro BS represented by player $i$, the CSB values of the pico BSs represented by player $i$, and the way of performing downlink scheduling.

Suppose user $u$ is attached to BS $j$ in the hexagon of player $i$ when player $i$ plays $s_{i}$, and the same user is attached to BS $k$ when player $i$ plays $s_{i}^{\prime}$, where BS $k$ is not necessarily in the hexagon of player $i$. At this point, user $u$ must be rescheduled to some PRBs offered by BS $k$, otherwise $V_{j}\left(s_{i}^{\prime}, \mathbf{s}_{-i}\right)$ becomes minus infinity. Such an outcome will prevent a player from changing its CSB values, which does not fulfill our objective of CSB optimization. Also, the PRBs that are assigned to user $u$ when player $i$ plays $s_{i}$ becomes unused when player $i$ plays $s_{i}^{\prime}$. These unused PRBs can be assigned to other users in order to improve the payoff function of player $i$. Therefore, it is necessary for a strategy of player $i$ to provide not only the scheduling of the BSs in player $i$ but also the scheduling of BSs in $\mathcal{N}_{i}^{\text {Att }}$, so that a strategy that changes user attachment patterns can have the chance of being a best/better response.

Let $\Gamma_{\varphi}^{\mathrm{eICIC}} \triangleq\left\langle\mathcal{L},\left\{\mathcal{S}_{i}^{\mathrm{eICIC}}: i \in \mathcal{L}\right\},\left\{V_{i},: i \in \mathcal{L}\right\}\right\rangle$ be the exact potential game for eICIC optimization using scheduler $\varphi$, where $\mathcal{S}_{i}^{\mathrm{eICIC}}$ denotes the set of strategies of player $i$ when eICIC optimization is performed. We have

$$
\mathcal{S}_{i}^{\mathrm{eICIC}}=\mathcal{A} \times \underbrace{\mathcal{C} \times \mathcal{C} \times \cdots \times \mathcal{C}}_{|i|-1 \text { times }} \times \varphi\left(\mathcal{N}_{i}^{\mathrm{Att}}\right),
$$

where $|i|$ is the number of BSs in the hexagon of player $i$, $|i|-1$ is the number of pico BSs in in the hexagon of player $i$, and $\varphi\left(\mathcal{N}_{i}^{\text {Att }}\right)$ gives the scheduling decision of the BSs in $\mathcal{N}_{i}^{\text {Att }}$ using scheduler $\varphi$.

The best response dynamics solves an exact potential game by iteratively finding the strategies that maximize the payoff functions of the players selected in each iteration. From the definition in (12), we can see that the size of the strategy 
set for eICIC optimization scales up quickly as the number of pico BSs in a hexagon increases. In order to reduce the complexity in each iteration of the best response dynamics, we use better response dynamics where only one pico BS's CSB value will be optimized in each iteration. Although, in general, better response dynamics cannot improve the payoff function of a player as much as best response dynamics does, better response dynamics also reaches a Nash equilibrium [20]. We therefore propose the following eICIC optimization algorithm based on exact potential game formulation:

I-1. Randomly select a player $i$ from $\mathcal{L}$.

I-2. Randomly select a pico BS $p$ in the set of BSs represented by player $i$.

I-3. Denote the macro BS in the hexagon of player $i$ as $m$. For all possible elements in $\mathcal{A} \times \mathcal{C}$, perform scheduling for all BSs in $\mathcal{N}_{i}^{\text {Att }}$ using scheduler $\varphi$ and evaluate $V_{i}$. Select the element in $\mathcal{A} \times \mathcal{C}$ that maximizes $V_{i}$.

I-4. Repeat the above steps until some stopping criterion is met.

The objective function in (4) will be improved when the above better response dynamic is carried out, because the aggregate utility of $\Gamma^{\mathrm{eICIC}}$ improves as a result of improved payoff function of each selected player during the better response dynamic. Therefore, the steps from I-1 to I-4 optimize (4) heuristically.

\section{B. The strategy sets and the algorithm for FeICIC}

Similar to the eICIC optimization, the strategy of a player should contain the RP-ABS pattern of the macro BS in the selected player, the CSB pattern of a randomly selected pico BS in the hexagon of the selected player, and the scheduling decision of the BSs in $\mathcal{N}_{i}^{\text {Att }}$. On the other hand, because in FeICIC optimization, the transmission power level in a $\mathrm{RP}-\mathrm{ABS}$ can take a fractional value, it is impossible to exhaustively search all possible transmission power levels for each RP-ABS.

Let $\tau(b) \in \hat{\mathcal{A}}$, and assume that macro BS $m$ belongs to player $i$ which is chosen to perform FeICIC optimization. Let $u$ be the index of the user who occupies PRB $b$ from macro BS $m$, and let $\mathcal{U}_{m, b}^{\mathrm{IF}}$ be the set of users who are interfered by the transmission of macro BS $m$ and who are using the $b$-th PRBs offered by their respective serving BSs. Fixing macro BS $m$ 's transmission power on PRBs other than $b$, we optimize macro BS $m$ 's transmission power on PRB $b$ by solving the following problem

\section{Powerallocation}

$$
\begin{aligned}
\underset{\hat{\boldsymbol{\alpha}}_{m}(b)}{\operatorname{maximize}} & w_{u} \ln \left(\log _{2}\left(1+\operatorname{SINR}_{u, b}^{m}\right)+r_{u}^{-b}\right) \\
& +\sum_{v \in \mathcal{U}_{m, b}^{\mathrm{IF}}}\left[w_{v} \ln \left(\log _{2}\left(1+\operatorname{SINR}_{v, b}^{j}\right)+r_{v}^{-b}\right)\right],
\end{aligned}
$$

subject to $0 \leq \hat{\boldsymbol{\alpha}}_{m}(b) \leq 1$,

where $r_{u}^{-b}$ gives user $u$ 's rate obtained from PRBs other than $b$ in case user $u$ has been allocated to more than one PRB and

$$
\operatorname{SINR}_{v, b}^{j}=\frac{h_{v, b}^{j} \cdot P_{j, v, b}^{\mathrm{Rx}}}{\hat{\boldsymbol{\alpha}}_{m}(b) \cdot P_{m, v}^{\mathrm{Rx}}+P_{\mathcal{I}_{p} \backslash\{m\}, v, b}^{\mathrm{IF}}+N_{0} \cdot W},
$$

where $P_{j, v, b}^{\mathrm{Rx}}$ is the received signal power of user $v$ at PRB $b$ from its serving $\mathrm{BS} j$ and $P_{m, v}^{\mathrm{Rx}}$ gives the interference power from macro BS $m$ to user $v$ when macro BS $m$ is transmitting at its maximum power. Note that the index $j$ in (14) is an element from $\mathcal{N}_{i}$, where $i$ is the index of the chosen player. Also, without loss of generality, we assume that $P_{j, v, b}^{\mathrm{Rx}}>0$, since we can remove user $v$ from $\mathcal{U}_{m, b}^{\mathrm{IF}}$ if $P_{j, v, b}^{\mathrm{Rx}}=0$.

The objective function of (13) is chosen to be in line with the objective function of (5) so that when (13) is optimized the objective function of (5) will also increase. Also, all variables in (13) are known except $\hat{\boldsymbol{\alpha}}_{m}(b)$. The next theorem shows the nature of the objective function of (13).

Theorem 3. Equation (13a) is the difference between two convex functions, where the two convex functions are $\sum_{v \in \mathcal{U}_{m, b}^{\mathrm{IF}}}\left[w_{v} \ln \left(\log _{2}\left(1+\operatorname{SINR}_{v, b}^{j}\right)+r_{v}^{-b}\right)\right]$ and $-w_{u} \ln \left(\log _{2}\left(1+\operatorname{SINR}_{u, b}^{m}\right)+r_{u}^{-b}\right)$.

Proof: See Appendix D.

Because of Theorem 3, (13) can be solved by the convexconcave procedure (CCP) which converges to a stationary point [21]. Let $\phi_{1}\left(\hat{\boldsymbol{\alpha}}_{m}(b)\right) \triangleq w_{u} \ln \left(\log _{2}\left(1+\operatorname{SINR}_{u, b}^{i}\right)+r_{u}^{-b}\right)$ and $\phi_{2}\left(\hat{\boldsymbol{\alpha}}_{m}(b)\right) \triangleq \sum_{v \in \mathcal{U}_{m, b}^{\mathrm{IF}}}\left[w_{v} \ln \left(\log _{2}\left(1+\operatorname{SINR}_{v, b}^{j}\right)+\right.\right.$ $\left.\left.r_{v}^{-b}\right)\right]$. Also, denote $\hat{\boldsymbol{\alpha}}_{m}^{\zeta}(b)$ as the value of $\hat{\boldsymbol{\alpha}}_{m}(b)$ in the $\zeta$ th iteration in the CCP. The CCP algorithm is described in Algorithm 1, where $\phi_{2}^{\prime}\left(\hat{\boldsymbol{\alpha}}_{m}(b)\right)$ is the first derivative of $\phi_{2}$ w.r.t. $\hat{\boldsymbol{\alpha}}_{m}(b)$ and it is given in (15) at the top of the next page. Note that step 4 in Algorithm 1 involves solving a convex problem which can be easily solved by standard software tools.

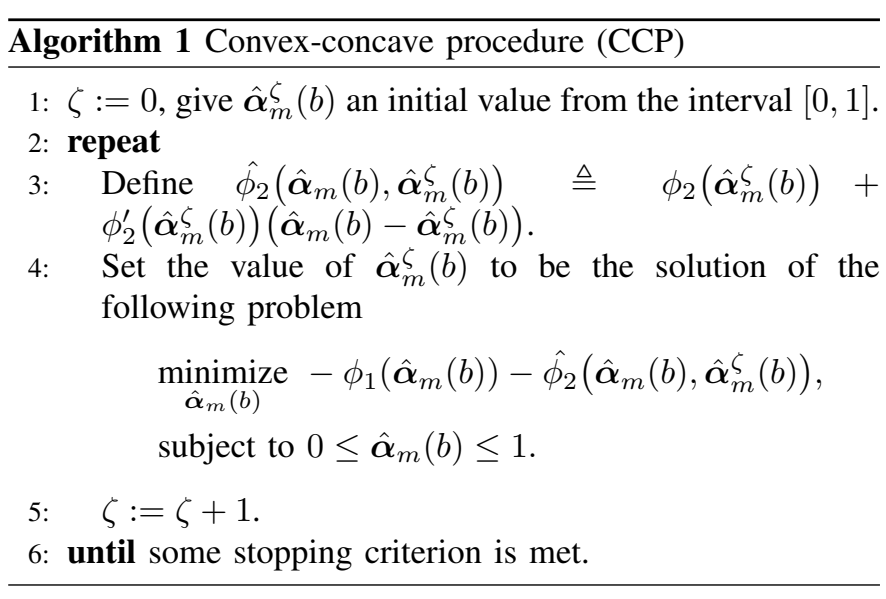

We are now ready to describe the FeICIC optimization algorithm based on the exact potential game formulation:

II-1. Randomly select a player $i$ from $\mathcal{L}$. Denote the macro BS in player $i$ as $m$.

II-2. Randomly select a pico BS $p$ from the set of BSs represented by player $i$.

II-3. For each possible CSB values of pico BS $p$,

a) Perform scheduling for all stations in $\mathcal{N}_{i}^{\text {Att }}$ using scheduler $\varphi$, assuming that it transmits at full power on all PRBs.

b) For each element in $\{b \mid \tau(b) \in \hat{\mathcal{A}}\}$, perform power optimization on macro BS $m$ 's transmission power by solving (13). 
$\phi_{2}^{\prime}\left(\hat{\boldsymbol{\alpha}}_{m}(b)\right)=-\sum_{v \in \mathcal{U}_{m, b}^{\mathrm{IF}}} \frac{\rho_{1, v} \rho_{2, v} w_{v}}{\left(\rho_{2, v} \hat{\boldsymbol{\alpha}}_{m}(b)+\rho_{3, v}\right)\left(\rho_{2, v} \hat{\boldsymbol{\alpha}}_{m}(b)+\rho_{3, v}+\rho_{1, v}\right)\left(\ln \left(1+\frac{\rho_{1, v}}{\rho_{2, v} \hat{\boldsymbol{\alpha}}_{m}(b)+\rho_{3, v}}\right)+\ln (2) r_{v}^{-b}\right)}$,

where $\rho_{1, v} \triangleq h_{u, b}^{j} P_{j, u, b}^{\mathrm{Rx}}, \rho_{2, v} \triangleq P_{m, u}^{\mathrm{Rx}}$, and $\rho_{3, v} \triangleq P_{\mathcal{I}_{p} \backslash\{m\}, u, b}^{\mathrm{IF}}+N_{0} W$.

c) Perform scheduling for all stations in $\mathcal{N}_{i}^{\text {Att }}$ using the scheduler $\varphi$ and evaluate $V_{i}$.

II-4. Select the strategy of player $i$ that maximizes $V_{i}$.

II-5. Repeat the above steps until some stopping criterion is met.

The steps from II-1 to II-5 optimize (5) heuristically, because the aggregate utility of $\Gamma^{\mathrm{FeICIC}}$ improves as the game is being played.

\section{Implementation in practice}

In LTE-A systems, a user's association is determined by the received signal strength and the offset value (i.e., CSB value in our context) from each of the candidate BSs. The offset values are stored in the system information blocks (SIBs) which are defined and broadcast to the users by the evolved universal terrestrial radio access network (E-UTRAN), i.e., by the BSs [22, Chapters 2 and 3]. A user continuously measures the channel conditions of its nearby BSs and reports these measurements to its serving BS. When a BS offers ABSs, a user served by the BS may find if a nearby pico BS has a better channel condition than its serving BS. Such information can be utilized by the serving BS to decide whether the CSB values should be updated so that a handover can be performed.

After accessing the interference situation of its users, a BS may request a neighboring $\mathrm{BS}$ for $\mathrm{ABSs}$ using an "Invoke Indication" message via the X2 interface. The BS that receives such a request may then configure its $\mathrm{ABS}$ pattern and inform its neighboring BSs such that the latter may perform scheduling based on the new ABS pattern [22, Chapters 31]. Also, BSs can adjust and coordinate the ABS patterns based on the "ABS Status" messages exchanged among them.

We can see that the LTE-A standards have prescribed signaling that allows FeICIC/eICIC optimizations to be carried out in a distributed manner. Via the signaling from neighboring BSs and the measurement reports from the users, a BS is able to know the interference situations of its users and the users served by nearby BSs. A BS can then decide how to adjust ABS/RP-ABS and CSB patterns for performance optimization.

The realization of the distributed optimizations is a design issue which is not standardized. Our proposed game theoretic framework provides distributed algorithms for eICIC/FeICIC optimizations and can be supported by the existing LTE-A standards. More specifically, the better response dynamics for eICIC/FeICIC optimizations are in the spirit of distributed optimization, since each player uses only local information to drive the overall system to optimality. In particular, each player is able to evaluate the impact of his strategy on his neighboring player's utilities. All this is possible thanks to the availability of the aforementioned signaling over the X2 interface.

\section{DOWNLINK SCHEDULERS}

We now present the downlink schedulers that can be the potential candidates for $\varphi$.

\section{A. Round-Robin (RR) Scheduler}

When using the RR scheduler, the available PRBs of a BS are allocated to the associated users in turns. For example, suppose a BS has five available PRBs labeled as $\mathrm{PRB}_{1}, \mathrm{PRB}_{2}$, $\ldots$, and $\mathrm{PRB}_{5}$, and two users are associated with the BS, then user 1 will get $\mathrm{PRB}_{1}, \mathrm{PRB}_{3}$, and $\mathrm{PRB}_{5}$, and user 2 will get $\mathrm{PRB}_{2}$ and $\mathrm{PRB}_{4}$. Note that a macro BS's PRBs that are ABSs will not be allocated to any user.

\section{B. PF Scheduler}

The $b$-th PRB of a BS will be allocated to the following user [22]:

$$
\widehat{u}_{b} \triangleq \arg \max _{u \in \mathcal{U}_{i}} \frac{r_{u, b}}{\bar{r}_{u}(\tau(b))},
$$

where $\tau(b)$ gives the subframe index of the $b$-th PRB and the underlying assumption is that subframe $\tau(b)$ is not an ABS, $b \in\left[1, N_{B}\right]$, and $\bar{r}_{u}(t)$ is the long-term average throughput of user $u$ in subframe $\tau(b)$ which is calculated as:

$$
\begin{aligned}
& \bar{r}_{u}(\tau(b))=\left(1-\frac{1}{t_{c}}\right) \bar{r}_{u}(\tau(b)-1) \\
& +\frac{1}{t_{c}} \sum_{\{\widetilde{b} \mid \tau(\widetilde{b})=\tau(b)\}} r_{u, \widetilde{b}} \cdot \mathbf{1}\left\{\widehat{u}_{b}=u\right\} .
\end{aligned}
$$

In (17), $t_{c}$ is the time window which is a design parameter and $\mathbf{1}\{\cdot\}$ is the indicator function. The performance of this scheduler has been evaluated in several scenarios; see [23].

\section{Convex Scheduler}

Given a strategy of player $i$, we wish to maximize the utility function of the players in $\mathcal{N}_{i}$ as defined in (8) subject to the constraints $(4 b),(4 c)$ and $(4 d)$. This problem is the same as (6) and it is, unfortunately, NP-hard as stated in Theorem 1.

On the other hand, we can relax the binary constraint in (6) to reduce the complexity of solving the problem. For example, considering pico BS $p$, we can relax the integer constraint in (4d) and formulate the following problem

\section{PRB-Allocation-RELAXED}

$$
\begin{gathered}
\operatorname{maximize} \sum_{u \in \mathcal{U}_{p}} w_{u} \cdot \ln \sum_{b=1}^{N_{B}}\left(\widetilde{x_{u, b}} \cdot r_{u, b}\right), \\
\text { subject to } 0 \leq \widetilde{x_{u, b}} \leq 1, \forall u \in \mathcal{U}_{p}, b \in\left[1, N_{B}\right], \\
\sum_{u \in \mathcal{U}_{p}} \widetilde{x_{u, b}}=1, b \in\left[1, N_{B}\right] .
\end{gathered}
$$

In (18), $\widetilde{x_{u, b}}$ represents the fraction of PRB $b$ allocated to user $u$. We make the following observations:

(a) The objective function of (18) is concave. To see this, notice that $\ln \sum_{b=1}^{N_{B}}\left(\widetilde{x_{u, b}} \cdot r_{u, b}\right)$ is a concave function 
of a linear combination of $\left\{\widetilde{x_{u, b}} \mid b \in\left[1, N_{B}\right]\right\}$. Therefore, the objective function of RELAXEDALLOCATION is also concave because it is a nonnegative summation of concave functions [24].

(b) The constraints of (18) are linear.

As a result, (18) is a concave optimization problem, and it can be solved by using standard convex optimization solvers. Let the matrix $\mathbf{X}^{\text {Relaxed }}$ be the solution to (18), where its $(u, b)$ th entry, $\mathbf{X}_{u, b}^{\text {Relaxed }}$, gives the fraction of the $b$-th PRB that is allocated to user $u$. To get an allocation pattern that satisfies the constraints of MAXPFUtiLity, we need to quantize $\mathbf{X}^{\text {Relaxed }}$. Also, we need to make sure that every user gets at least one PRB after quantization, because the utility function in (8) evaluates to minus infinity if no PRB is allocated to a user, which contradicts with the goal of maximizing the utility function. The quantization can be done in the following steps:

1) For each column of $X^{\text {Relaxed }}$, set the largest element in the column to one and other elements to zeros. Denote the resultant matrix as $\mathbf{X}^{\text {Quan. }}$.

2) If there exists a zero row in $X^{\text {Quan.: }}$

a) Denote all columns of $\mathbf{X}^{\text {Quan. }}$ as free columns.

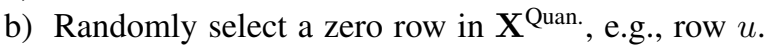

c) Let $\mathbf{X}_{u, b}$ be the largest element in row $u$ of $\mathbf{X}^{\text {Relaxed }}$ where column $b$ is still a free column in $\mathbf{X}^{\text {Quan. }}$. Set $\mathbf{X}_{u, b}^{\text {Quan. }}$ to one and every other element in column $b$ of $\mathbf{X}^{\text {Quan. }}$ to zeros.

d) Remove column $b$ from the free column list. Repeat steps b) and c) if there still exists a zero row in $\mathbf{X}^{\text {Quan. }}$.

The above quantization ensures that the PRB allocation constraints in (4) and (4) are satisfied, and at the same time each user gets at least one PRB.

The scheduler for a macro BS is similar and therefore its details are omitted for brevity. The only difference is that PRBs that are configured as ABSs are not allocated to any user.

\section{Cake-Cutting Scheduler}

We now present a method that solves (18). We use the fact that the solution to (18) leads to a price equilibrium to the following PRICEEQUilibrium problem [25, Chapter 8.5]:

PriceEquilibrium. Let $r_{u, b}$ be nonnegative real numbers, where $u \in \mathcal{U}_{i}, b \in\left[1, N_{B}\right]$. The real vector $\left(\nu_{1}, \nu_{2}, \ldots, \nu_{N_{B}}\right)$ is called an equilibrium price vector and the nonnegative real vectors $\left\{\left(\widetilde{x_{u, 1}}, \widetilde{x_{u, 2}}, \ldots, \widetilde{x_{u, N_{B}}}\right) \mid u \in \mathcal{U}_{i}\right\}$ are called equilibrium bundles, if

$$
\begin{array}{rc}
\left\{\widetilde{x_{u, b}} \mid \forall b\right\} \text { maximize } & \sum_{b} \nu_{b}=\sum_{u} w_{u}, \\
\text { subject to } & \left.\sum_{b} \nu_{b} \cdot \widetilde{x_{u, b}} \cdot r_{u, b}\right), \forall u, \\
& \sum_{u} \widetilde{x_{u, b}}=1, \forall b .
\end{array}
$$

The intuition for PRICEEQUILIBRIUM is as follows. There are $N_{B}$ goods in the market each with price $\nu_{b}$, where $b \in$ $\left[1, N_{B}\right]$. User $u$ has budget $w_{u}$ and he is allowed to buy a nonnegative portion of any good. $r_{u, b}$ gives the utility of the $b$-th good to user $u$. A price equilibrium is the set of prices of the goods so that all users spend all their budgets, all goods are sold out, and under these conditions all users maximize their own utilities.

Let $\left\{x_{u, b}^{*} \mid \forall u, b\right\}$ be the solution to (18). It is proved in [18] that a price equilibrium of PRICEEQUILIBRIUM gives an optimal solution to (18).

Theorem 4. A solution of PRICEEQUILIBRIUM gives an optimal solution to (18).

Proof: See [18].

The PriceEquilibrium problem can be solved by the algorithm proposed in [26]. The algorithm works by iteratively adjusting the prices of the goods and assumes that a user only buys the goods that have the largest utilities to him. Each iteration of the algorithm involves solving a max flow problem in a single-source single-sink directed graph where the edges are weighted, and therefore an iteration takes polynomial time. The algorithm terminates within finite iterations, though [26] does not provide an upper bound on the number of iterations. In the simulation section, we will compare the run time of the cake-cutting PF scheduler to that of the convex PF scheduler.

\section{Simulation Results}

We perform simulation studies on FeICIC and eICIC optimizations by randomly generating $100 \mathrm{HetNet}$ topologies and then averaging the performance indicators from all the topologies. In the center cluster of each topology, a number of pico BSs and 20 users are placed inside each hexagon in the center cluster, where the pico BSs are randomly placed. Moreover, in each hexagon in the center cluster, 10 users are randomly placed within 100 meters of the pico BSs in the same hexagon ${ }^{3}$. The distances between different BSs and the distances between BSs and users are constrained by the minimum distance requirements as specified in Table II. The six surrounding clusters of the center cluster are exact copies of the center cluster. Other parameters regarding the generation of a random HetNet are also shown in Table II. We assume that the users are static. Also, each PRB experiences independent Rayleigh fading with variance 1 . The shadow fading in $d B$ from a BS to a user is calculated by adding a common shadowing value and a random shadowing value and then dividing the sum by $\sqrt{2}$, where both shadowing values are generated according to log-normal distribution [27] ${ }^{4}$.

The parameters of the problems (4) and (5) for simulations are configured as follows. The weighting factors of all users are set to be 1, i.e., $w_{u}=1$ for all $u$. $N_{T}$ is set to be 10 and $N_{F}$ is set to be 3. Fig. 2 shows all the possible ABS patterns. Also, the CSB values that a pico BS can adopt are given in Table II. For conciseness, in the rest of the figures, we use "Nil" to represent the case where neither eICIC nor FeICIC is carried out, "Exact" to represent the case where $\mathcal{N}_{i}^{\mathrm{IF}}=i \cup \mathcal{N}(i, 1)$, and "Non-exact" to represent $\mathcal{N}_{i}^{\mathrm{IF}}=i \cup \mathcal{N}(i, 1) \cup \mathcal{N}(i, 2) \cup \mathcal{N}(i, 3)$. Moreover, we use the terms cell and hexagon interchangeably.

\footnotetext{
${ }^{3}$ If there exists more than one pico BS, then the 10 users are equally divided into a number of groups which is the same as the number of pico BSs, and one and only one group of users are randomly placed near a pico BS.

${ }^{4}$ This is to create correlations among shadow fading.

${ }^{5} \mathrm{Min}$. dist. stands for minimum distance.
} 


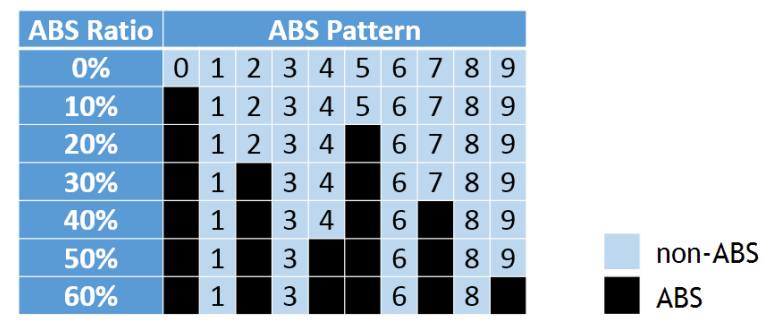

Fig. 2: ABS patterns that can be chosen by a macro BS.

TABLE II: Parameters for generating HetNet topologies [27].

\begin{tabular}{|c|c|}
\hline Parameter & Value \\
\hline Inter-macro-BS distance & $1000 \mathrm{~m}$ \\
\hline Min. dist. ${ }^{5}$ from macro BS to user & $35 \mathrm{~m}$ \\
\hline Min. dist. from pico BS to user & $10 \mathrm{~m}$ \\
\hline Min. dist. from macro BS to pico BS & $75 \mathrm{~m}$ \\
\hline Antenna per site & Omnidirectional $\times 1$ \\
\hline Macro BS power & $40 \mathrm{~W}$ \\
\hline Pico BS Power & $1 \mathrm{~W}$ \\
\hline Noise density & $9 \mathrm{~dB} / \mathrm{Hz}$ \\
\hline Noise figure & $1 \mathrm{~ms}$ \\
\hline Duration per subframe & $180 \mathrm{kHz}$ \\
\hline Bandwidth per RB & $10 \mathrm{~dB}$ \\
\hline CSB values & $\mathcal{C}:=\{0,3,6,9,15\} \mathrm{dB}$ \\
\hline Log-normal shadowing & \\
\hline standard deviation & $128.1+37.6 \log _{10} d, d$ in $\mathrm{km}$ \\
\hline Path loss from macro BS to user & $140.7+36.7 \log _{10} d, d$ in $\mathrm{km}$ \\
\hline Path loss from pico BS to user
\end{tabular}

Fig. 3 shows the EE of different optimization schemes in the center cluster, where the EE is calculated as the number of transmitted bits divided by the transmission energy. We can see that eICIC optimization largely improves the EE and FeICIC optimization further enhances the EE. Specifically, when the $\mathrm{PF}$ scheduler is used and there are three pico BSs in each hexagon, eICIC can offer about $64 \%$ improvement compared to the no-optimization case and FeICIC can offer about $92 \%$ compared to the no-optimization case. Moreover, compared to the no-optimization case, eICIC and FeICIC can offer more gain on EE when there are more pico BSs in each hexagon.

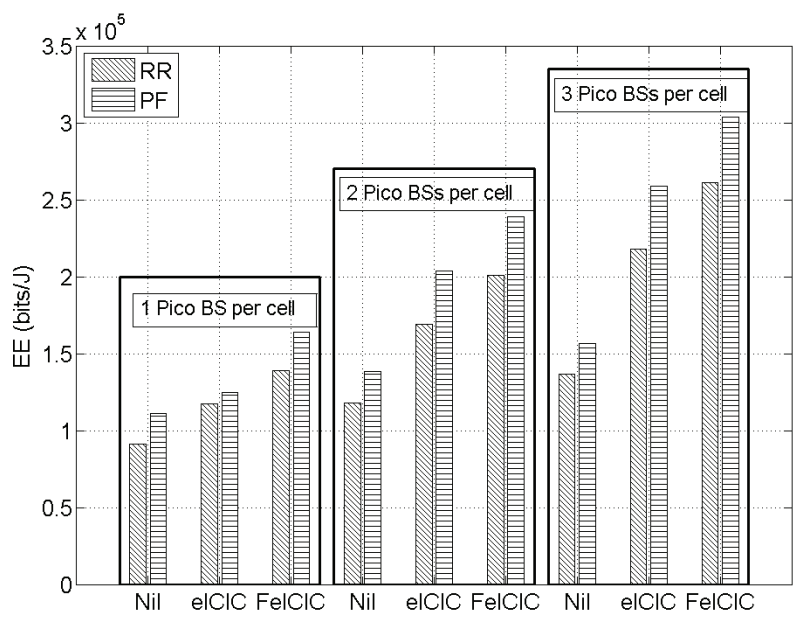

Fig. 3: EE of the center cluster for various topology settings.

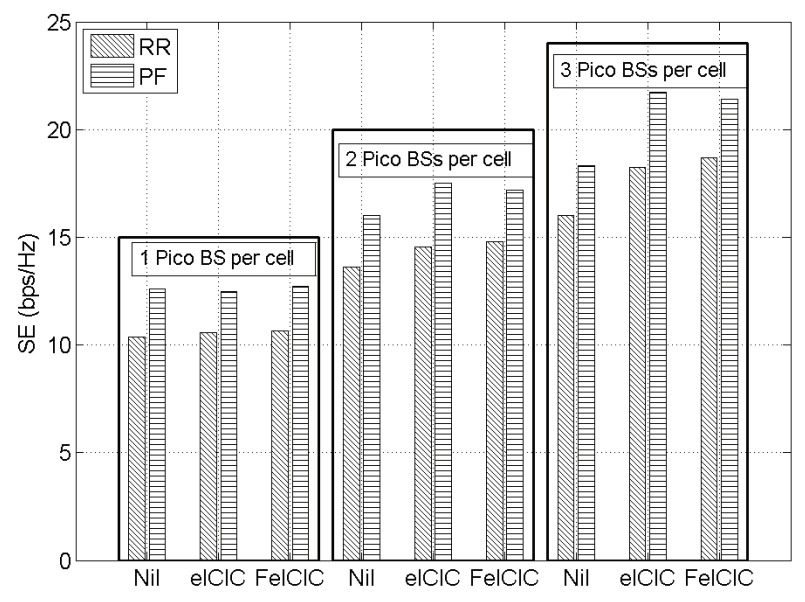

Fig. 4: SE of the center cluster for various topology settings.

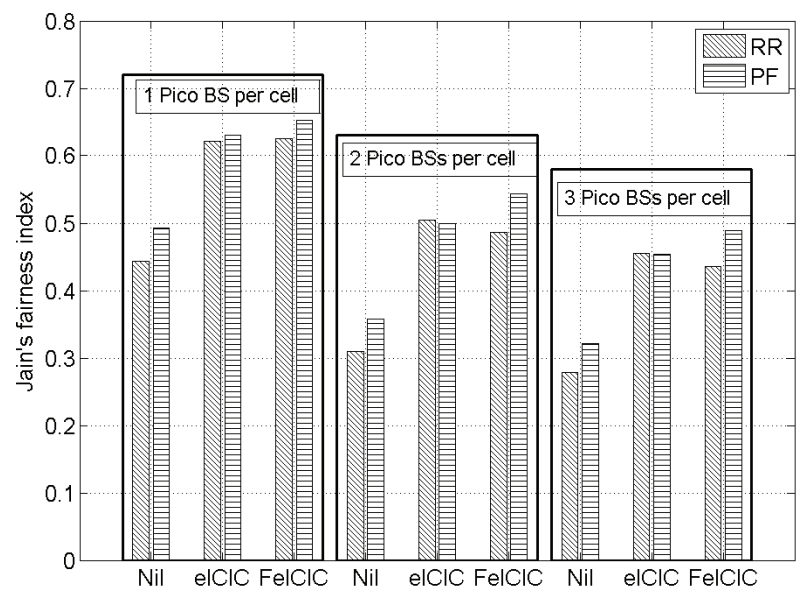

Fig. 5: Jain's fairness indices of the users' achieved rates in the center cluster for various topology settings.

Fig. 4 shows the SE of different optimization schemes in the center cluster, where the SE is defined as the average transmitted bits per second per $\mathrm{Hz}$ of all allocated PRB. Although the SE after FeICIC optimization using PF scheduler may be slightly (around 1\% in general) less than the SE after eICIC optimization, FeICIC optimization should still be treated as a better scheme than eICIC optimization because compared to eICIC optimization, FeICIC optimization offers significant gain on EE, offers better fairness when PF scheduler is used as shown in Fig. 5, and offers better worst 5\% user's achieved rates as shown in Fig. 6.

Fig. 6 plots the cumulative distribution function (CDF) of the users' achieved rates which are in the worst 5\% range of all users' achieved rates ${ }^{6}$, where the PF scheduler is used and each hexagon has one pico BS. We can see that eICIC optimization can improve the median value of the worst $5 \%$ users' achieved rates by about $30 \%$, and FeICIC can further

\footnotetext{
${ }^{6}$ This is also commonly referred to as the cell-edge throughput.
} 


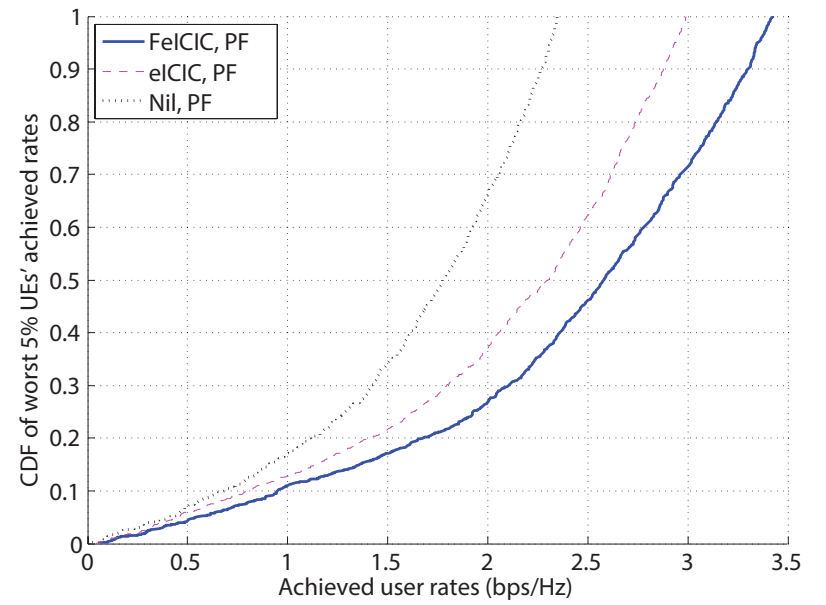

Fig. 6: The cumulative distribution function (CDF) of the worst $5 \%$ users' achieved rates in the center cluster, where the PF scheduler is used and each hexagon has one pico BS.

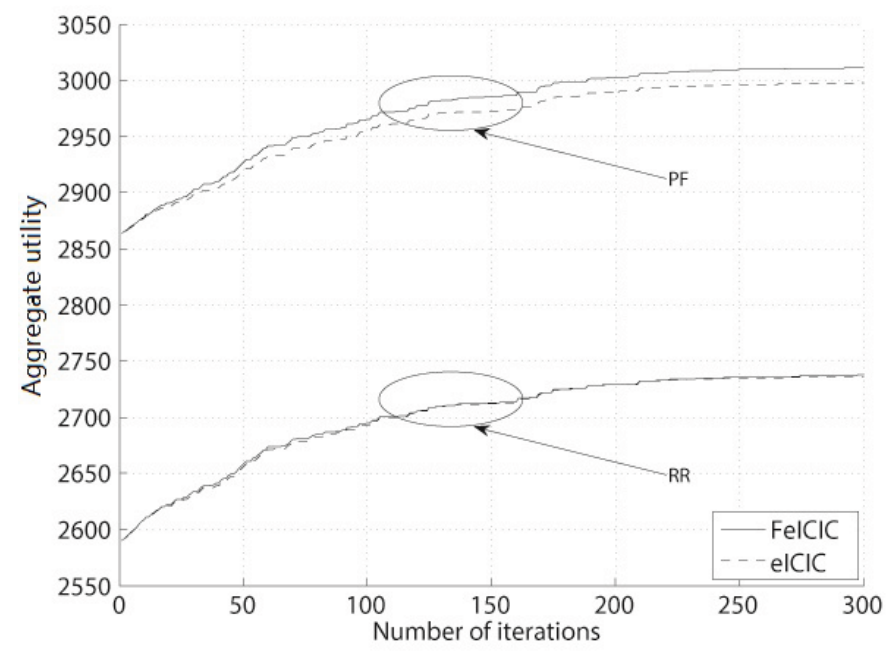

Fig. 7: Aggregate utilities of the games $\Gamma^{\mathrm{FeICIC}}$ and $\Gamma^{\mathrm{eICIC}}$, where there are 3 pico BSs in each hexagon.

improve the median value by about $15 \%$.

Fig. 7 plots the averaged global utilities of the games $\Gamma^{\text {FeICIC }}$ and $\Gamma^{\mathrm{eICIC}}$ when both RR and PF schedulers are used, where three pico BSs are placed in each hexagon. We can see that the better response dynamics proposed in Sections V-A and V-B can optimize the problems (4) and (5) heuristically because the global utilities increase as the games are being played.

The results in Fig. 3 to Fig. 7 are obtained when player $i$ only interferes near-by users, i.e., $\mathcal{N}_{i}^{\mathrm{IF}}=i \cup \mathcal{N}(i, 1)$. In reality, however, the interference from a BS can reach farther. Suppose we count the interference from BSs in $\bigcup_{n=0}^{4} \mathcal{N}\left(m_{u}, n\right)$ to user $u$ as all the interference user $u$ suffers from, where no ABS/RP-ABS or CSB is applied. Table III summarizes the averaged interference power to a user from the neighboring cells. The definition of $\mathcal{N}_{i}^{\mathrm{IF}}=i \cup \mathcal{N}(i, 1)$ takes $95.38 \%$ of the total interference into account.

To see the impact of interference approximation, we compare the performance of the game theoretic optimization schemes when $\mathcal{N}_{i}^{\mathrm{IF}}=i \cup \mathcal{N}(i, 1)$ and $\mathcal{N}_{i}^{\mathrm{IF}}=i \cup \mathcal{N}(i, 1) \cup$
TABLE III: Average interference to user $u$ from neighboring BSs.

\begin{tabular}{|c|c|c|c|c|}
\hline & $\begin{array}{c}\mathcal{N}\left(m_{u}, 0\right) \\
\cup \mathcal{N}\left(m_{u}, 1\right)\end{array}$ & $\mathcal{N}\left(m_{u}, 2\right)$ & $\mathcal{N}\left(m_{u}, 3\right)$ & $\mathcal{N}\left(m_{u}, 4\right)$ \\
\hline $\begin{array}{l}\text { Interference } \\
\text { Power (dB) }\end{array}$ & -106.59 & -122.42 & -124.70 & -128.17 \\
\hline Percentage & $95.38 \%$ & $2.49 \%$ & $1.47 \%$ & $0.66 \%$ \\
\hline
\end{tabular}

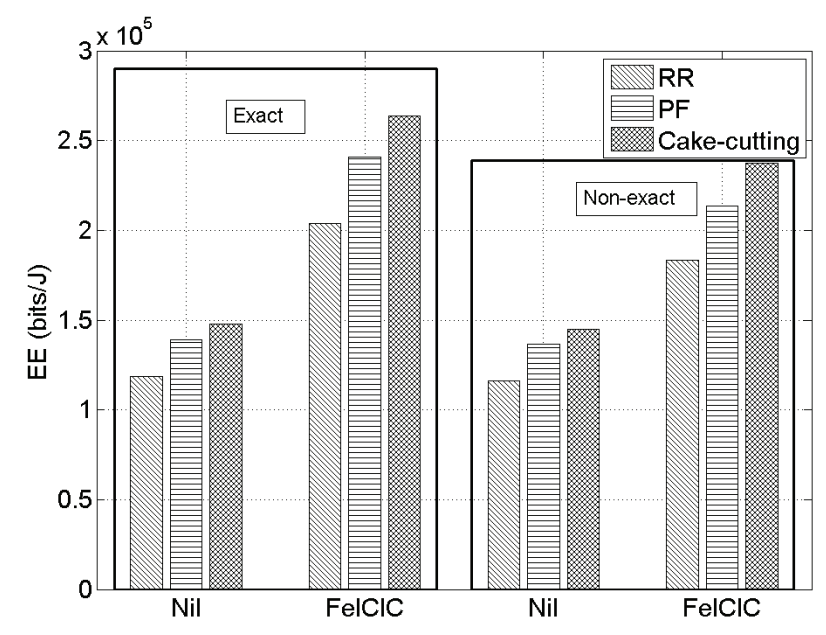

Fig. 8: EE when there are two pico BSs in each hexagon.

$\mathcal{N}(i, 2) \cup \mathcal{N}(i, 3)$, where $i$ is a player in the games $\Gamma^{\text {FeICIC }}$ and $\Gamma^{\mathrm{eICIC}}$. Note that when $\mathcal{N}_{i}^{\mathrm{IF}}=i \cup \mathcal{N}(i, 1) \cup \mathcal{N}(i, 2) \cup \mathcal{N}(i, 3)$, the games $\Gamma^{\mathrm{FeICIC}}$ and $\Gamma^{\mathrm{eICIC}}$ will no longer be exact potential games because $(11 \mathrm{c})$ is no longer true. For the scenario where there are two pico BSs in each hexagon, we plot the EE, the $\mathrm{SE}$, and the Jain's fairness indices after FeICIC optimization in Fig. 8, Fig. 9, and Fig. 10, respectively, where in those figures, "Exact" represent the case when $\mathcal{N}_{i}^{\mathrm{IF}}=i \cup \mathcal{N}(i, 1)$ and "Nonexact" represent the case when $\mathcal{N}_{i}^{\mathrm{IF}}=i \cup \mathcal{N}(i, 1) \cup \mathcal{N}(i, 2) \cup$ $\mathcal{N}(i, 3)$. We can see that although the performances of the optimization schemes are over-estimated by the assumption of $\mathcal{N}_{i}^{\mathrm{IF}}=i \cup \mathcal{N}(i, 1)$, the performance gains achieved by FeICIC compared to the no-optimization case is accurately predicted.

The performance of the cake-cutting scheduler is compared with RR scheduler and PF scheduler in Fig. 8, Fig. 9, and Fig. 10. We can observe that the cake-cutting scheduler can lead to better performance on EE, SE, and fairness compared to the other schedulers. Specifically, when FeICIC is performed, the cake-cutting scheduler has approximately an $11 \%$ gain on EE and approximately a $10 \%$ gain on SE.

Table IV shows the average MATLAB simulation run time for each macro BS to perform downlink scheduling using different schedulers, where it is assumed that no pico BS is present and each macro BS serves exactly 10 users. The MATLAB version is R2013a, and the simulation is performed on a laptop equipped with an Intel i5-4200U CPU using single thread. For the convex scheduler, we compare all the four available convex solvers in MATLAB's built-in function "fmincon", and we record the run time of the fastest solver. We observe that the scheduling decisions resulted from the convex scheduler are almost the same as those from the cake-cutting 


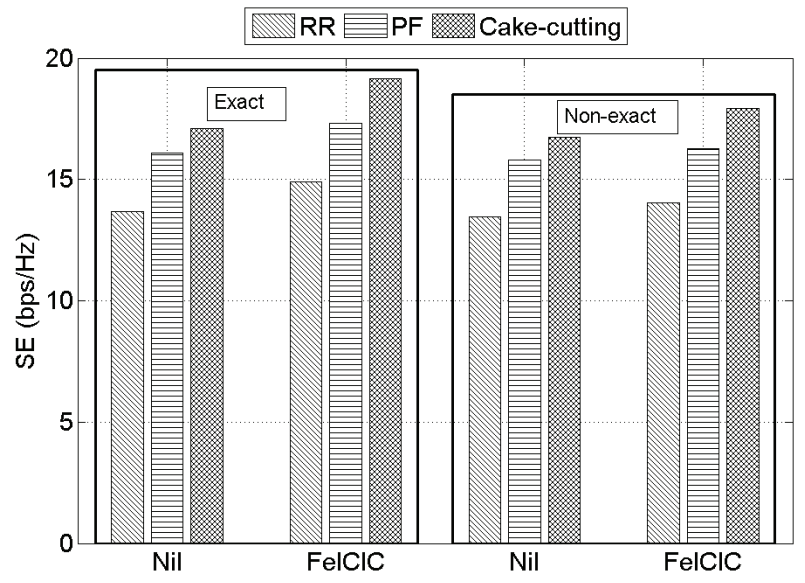

Fig. 9: SE when there are two pico BSs in each hexagon.

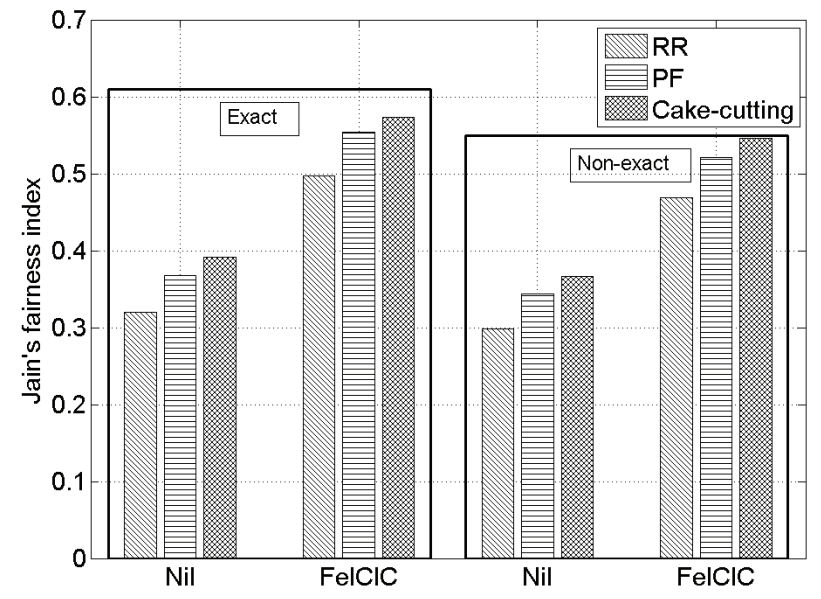

Fig. 10: Jain's fairness indices of users' achieved rates when there are two pico BSs in each hexagon.

scheduler, but the averaged run time of the convex scheduler is about 20 times as long as that of the cake-cutting scheduler. Although the PF scheduler and the RR scheduler run faster than the cake-cutting PF scheduler, the cake-cutting scheduler gives better performance in terms of $\mathrm{EE}, \mathrm{SE}$, and fairness in users' achieved rates.

\section{CONCLUSION}

In this paper, we have proposed distributed algorithms based on the exact potential game framework to optimize FeICIC and eICIC in LTE-A HetNets. Through simulation studies, we have

TABLE IV: Comparison on downlink schedulers' run time.

\begin{tabular}{|c|c|}
\hline Scheduler & Average Run Time per BS \\
\hline Convex & 7.5275 seconds \\
\hline Cake-cutting & 0.3860 seconds \\
\hline PF $t_{c}=5$ & 0.0045 seconds \\
\hline RR & 0.0018 seconds \\
\hline
\end{tabular}

demonstrated that eICIC optimization can improve the EE of the network by $64 \%$ while FeICIC optimization can improve the EE by about $92 \%$. In addition, FeICIC can offer better fairness in users' throughputs and can also yield significant cell-edge throughput gains compared to eICIC. Furthermore, we have shown that a cake-cutting algorithm can be used as a downlink scheduler to offer better EE, SE, and fairness among users compared to conventional PF schedulers while being much more computationally efficient than the conventional convex-solvers.

\section{ACKNOWLEDGEMENT}

The authors would like to thank Dr. Kenneth W. Shum for providing his software implementation of the cake-cutting algorithm. Also, the authors would like to thank the anonymous reviewers whose comments have helped the authors to improve the quality of the paper significantly. A part of the work was carried out when the first author was a visiting student at the Laboratory of Information, Networking and Communication Sciences (www.lincs.fr).

\section{APPENDIX A PROOF OF PROPOSITION 1}

Given a user $u$ :

1) Suppose $m_{u}=j$, where $j \in i$, then user $u$ can be associated with any BSs in $\mathcal{O}_{u}=i \cup \mathcal{N}(i, 0) \cup \mathcal{N}(i, 1)$ for any CSB values that the pico BSs represented by player $i$ takes.

2) Suppose $m_{u} \in \mathcal{N}(i, 1)$ and $g\left(u, \gamma_{\mathcal{O}_{u}}\right)=j$, where $j \in i$. If we change $\gamma_{\mathcal{O}_{u}}(j)$, then:

a) Depending on the CSB value of BS $i$, user $u$ can be associated with a BS in $\mathcal{N}(i, 2)$ because $\mathcal{N}(i, 2) \cap$ $\mathcal{O}_{u} \neq \emptyset$ and $i \subset \mathcal{O}_{u}$.

b) User $u$ cannot be associated with any BS in $\mathcal{N}(i, x)$, where $x \geq 3$. The reason is that $\mathcal{N}(i, x) \cap$ $\mathcal{O}_{u}=\emptyset$, for $x \geq 3$.

3) Suppose $m_{u} \in \mathcal{N}(i, x)$, where $x \geq 2$. In this case, the change of CSB values of player $i$ will not affect the association of user $u$ because player $i$ is not in $\mathcal{O}_{u}$.

Summarizing the above three scenarios, we can conclude that $\mathcal{N}_{i}^{\text {Att }}=i \cup \mathcal{N}(i, 0) \cup \mathcal{N}(i, 1) \cup \mathcal{N}(i, 2)$.

\section{APPENDIX B \\ ProOf OF PROPOSITION 2}

Suppose player $i$ changes its strategy from $s_{i}$ to $s_{i}^{\prime}$. We can easily see that if the difference between $s_{i}$ and $s_{i}^{\prime}$ includes the scheduling decision, then the scheduling change will only affect $U_{i}$. Therefore, we can decouple the effect of scheduling change and the following three cases are sufficient to determine which players' utility functions will be affected:

C-1. If $s_{i}$ and $s_{i}^{\prime}$ differs by the ABS/RP-ABS pattern only, then users who are located in the same hexagons as BSs in $\mathcal{N}_{i}^{\mathrm{IF}}$ will have their achieved rates changed, while these users can be attached to BSs in $i \cup \mathcal{N}(i, 1) \cup$ $\mathcal{N}(i, 2)=\mathcal{N}_{i}^{\text {Att }}$. Therefore, utilities of players in $\mathcal{N}_{i}^{\text {Att }}$ may be changed in this case. 


$$
f_{2, v}^{\prime \prime}\left(\hat{\boldsymbol{\alpha}}_{m}(b)\right)=\frac{\rho_{1, v} \rho_{2, v}^{2} w_{v}\left\{\rho_{1, v}\left[\left(\frac{2 \rho_{2, v} \hat{\boldsymbol{\alpha}}_{m}(b)+2 \rho_{3, v}}{\rho_{1, v}}+1\right) \ln \left(1+\frac{\rho_{1, v}}{\rho_{2, v} \hat{\boldsymbol{\alpha}}_{m}(b)+\rho_{3, v}}\right)-1\right]+\mu_{v}\right\}}{\left(\rho_{2, v} \hat{\boldsymbol{\alpha}}_{m}(b)+\rho_{3, v}\right)^{2}\left(\rho_{2, v} \hat{\boldsymbol{\alpha}}_{m}(b)+\rho_{3, v}+\rho_{1, v}\right)^{2}\left[\ln \left(1+\frac{\rho_{1, v}}{\rho_{2, v} \hat{\boldsymbol{\alpha}}_{m}(b)+\rho_{3, v}}\right)+\ln (2) r_{v}^{-b}\right]^{2}}
$$

C-2. If $s_{i}$ and $s_{i}^{\prime}$ differs by the CSB patterns only, and consequently user $u$ 's serving BS is changed from $n$ to $j$, then the utilities of the players where $\mathrm{BS} n$ and BS $j$ belong to will be changed and $m_{u} \in i \cup \mathcal{N}(i, 1)$. Moreover:

a) If $m_{u} \in i$, then $n \in \mathcal{O}_{u} \subset \mathcal{N}_{i}^{\text {Att }}$ and $j \in i \cup$ $\mathcal{N}(i, 1) \subset \mathcal{N}_{i}^{\text {Att }}$.

b) If $m_{u} \in \mathcal{N}(i, 1)$, then $\{n, j\} \in \mathcal{O}_{u} \subset i \cup \mathcal{N}(i, 1) \cup$ $\mathcal{N}(i, 2)$. In other words, $\{n, j\} \in \mathcal{N}_{i}^{\text {Att }}$.

C-3. Suppose $s_{i}$ and $s_{i}^{\prime}$ differs by both the ABS/RP-ABS and the CSB patterns, and consequently user $u$ 's serving BS is changed from $n$ to $j$. From the analysis of C-1, we know that users who are interfered by player $i$ can only be attached to BSs in $\mathcal{N}_{i}^{\text {Att }}$. From the analysis of C-2, we know that a user may only change its serving BS from a BS in $\mathcal{N}_{i}^{\text {Att }}$ to another BS in $\mathcal{N}_{i}^{\text {Att }}$. Therefore, the changes of ABS and CSB patterns will only affect the utilities of players in $\mathcal{N}_{i}^{\text {Att }}$.

Summarizing the above arguments, we can conclude that only $U_{j}$ can be changed if $s_{i}$ is changed to $s_{i}^{\prime}$, where $j \subset \mathcal{N}_{i}^{\text {Att }}$.

The statement that $\mathcal{N}_{i}=\mathcal{N}_{i}^{\text {Att }}$ given that $\mathcal{N}_{i}^{\mathrm{IF}}=i \cup \mathcal{N}(i, 1)$ is readily true by the definition of $\mathcal{N}_{i}$.

\section{APPENDIX C \\ PROOF OF PROPOSITION 3}

Suppose user $u$ is located in the same hexagon as BS $j$. Then it is possible that user $u$ is attached to a BS in $\mathcal{N}(i, 3)$ because $\mathcal{O}_{u} \cap \mathcal{N}(i, 3) \neq \emptyset$. This means that $\mathcal{N}(i, 3) \cap \mathcal{N}_{i} \neq \emptyset$. Because $\mathcal{N}_{i}^{\text {Att }} \cap \mathcal{N}(i, 3)=\emptyset$ and by definition $\mathcal{N}_{i}^{\text {Att }} \subseteq \mathcal{N}_{i}$, we conclude that $\mathcal{N}_{i}^{\text {Att }} \subset \mathcal{N}_{i}$.

\section{APPENDIX D}

\section{PROOF OF THEOREM 3}

For brevity of presentation, let $\rho \triangleq \frac{\operatorname{SINR}_{u, b}^{m}}{\hat{\boldsymbol{\alpha}}_{m}(b)}$. Also, note that $\operatorname{SINR}_{v, b}^{j}=\frac{\rho_{1, v}}{\hat{\boldsymbol{\alpha}}_{m}(b) \rho_{2, v}+\rho_{3, v}}$, where $\rho_{1, v}, \rho_{2, v}$, and $\rho_{3, v}$ are defined in (15). Then, (13a) can be rewritten as

$$
\begin{aligned}
& w_{u} \ln \left(\log _{2}\left(1+\hat{\boldsymbol{\alpha}}_{m}(b) \cdot \rho\right)+r_{u}^{-b}\right) \\
& +\sum_{v \in \mathcal{U}_{m, b}^{\mathbb{F}}}\left[w_{v} \ln \left(\log _{2}\left(1+\frac{\rho_{1, v}}{\hat{\boldsymbol{\alpha}}_{m}(b) \rho_{2, v}+\rho_{3, v}}\right)+r_{v}^{-b}\right)\right] \\
= & C+f_{1}\left(\hat{\boldsymbol{\alpha}}_{m}(b)\right)+\sum_{v \in \mathcal{U}_{m, b}^{\mathbb{F}}} f_{2, v}\left(\hat{\boldsymbol{\alpha}}_{m}(b)\right),
\end{aligned}
$$

where $C \triangleq \ln \left(\frac{1}{\ln (2)}\right)\left(w_{u}+\sum_{v \in \mathcal{U}_{m, b}^{\mathrm{IF}}} w_{v}\right)$ is a constant,

$$
f_{1}\left(\hat{\boldsymbol{\alpha}}_{m}(b)\right) \triangleq w_{u} \ln \left(\ln \left(1+\hat{\boldsymbol{\alpha}}_{m}(b) \cdot \rho\right)+\ln (2) r_{u}^{-b}\right),
$$

and

$$
f_{2, v}\left(\hat{\boldsymbol{\alpha}}_{m}(b)\right) \triangleq w_{v} \ln \left(\ln \left(1+\frac{\rho_{1, v}}{\hat{\boldsymbol{\alpha}}_{m}(b) \rho_{2, v}+\rho_{3, v}}\right)+\ln (2) r_{v}^{-b}\right) .
$$

Observe that $f_{1}\left(\hat{\boldsymbol{\alpha}}_{m}\right)$ is concave because $w_{u}$ is non-negative, $\ln \left(1+\hat{\boldsymbol{\alpha}}_{m}(b) \cdot \rho\right)+\ln (2) r_{u}^{-b}$ is concave and $\ln (\cdot)$ is a nondecreasing concave function [24, pp. 84].

Next, we argue that $f_{2, v}\left(\hat{\boldsymbol{\alpha}}_{m}(b)\right)$ is convex. The second derivative of $f_{2, v}\left(\hat{\boldsymbol{\alpha}}_{m}(b)\right)$ w.r.t. $\hat{\boldsymbol{\alpha}}_{m}(b)$ is given in (20), where $\mu_{v}=2 \ln (2) \rho_{2, v} r_{v}^{-b} \hat{\boldsymbol{\alpha}}_{m}(b)+\left(2 \ln (2) \rho_{3, v}+\ln (2) \rho_{1, v}\right) r_{v}^{-b} \geq$ 0 . We now argue that $f_{2, v}^{\prime \prime}\left(\hat{\boldsymbol{\alpha}}_{m}(b)\right)>0$. Let $q_{v} \triangleq$ $\frac{\rho_{1, v}}{\rho_{2, v} \hat{\boldsymbol{\alpha}}_{m}(b)+\rho_{3, v}}$, where $q_{v}>0$ by definition. Then,

$$
\begin{aligned}
& \rho_{1, v}\left[\left(\frac{2 \rho_{2, v} \hat{\boldsymbol{\alpha}}_{m}(b)+2 \rho_{3, v}}{\rho_{1, v}}+1\right) \ln \left(1+\frac{\rho_{1, v}}{\rho_{2, v} \hat{\boldsymbol{\alpha}}_{m}(b)+\rho_{3, v}}\right)-1\right] \\
= & \rho_{1, v} \frac{\left(2+q_{v}\right) \ln \left(1+q_{v}\right)-q_{v}}{q_{v}}>\rho_{1, v} \frac{\left(1+q_{v}\right) \ln \left(1+q_{v}\right)-q_{v}}{q_{v}}, \text { (24) }
\end{aligned}
$$

where (24) is true because $\ln \left(1+q_{v}\right)>0$. Then, notice that (24) is positive because $\rho_{1, v}>0$ by definition and $\left(1+q_{v}\right) \ln \left(1+q_{v}\right)-q_{v}>0$ for $q_{v}>0$ for the following reasons:

1) $\lim _{q_{v} \rightarrow 0^{+}}\left(1+q_{v}\right) \ln \left(1+q_{v}\right)-q_{v}=0$.

2) The derivative of $\left(1+q_{v}\right) \ln \left(1+q_{v}\right)-q_{v}$ w.r.t. $q_{v}$ is $\ln \left(1+q_{v}\right)$ which is larger than zero for $q_{v}>0$, meaning that $\left(1+q_{v}\right) \ln \left(1+q_{v}\right)-q_{v}$ is an increasing function of $q_{v}$ when $q_{v}>0$.

Therefore, $f_{2, v}^{\prime \prime}\left(\hat{\boldsymbol{\alpha}}_{m}(b)\right)>0$ because (24) is positive and the terms $\rho_{1, v}, \rho_{2, v}, \rho_{3, v}$, and $w_{v}$ are all positive. The fact that $f_{2, v}^{\prime \prime}\left(\hat{\boldsymbol{\alpha}}_{m}(b)\right)>0$ implies that $f_{2, v}\left(\hat{\boldsymbol{\alpha}}_{m}(b)\right)$ is convex $^{7}$ [24].

From (21), (22), and (23), the objective function of (13) can be rewritten as

$$
C+\sum_{v \in \mathcal{U}_{m, b}^{\mathbb{F}}} f_{2, v}\left(\hat{\boldsymbol{\alpha}}_{m}(b)\right)-\left(-f_{1}\left(\hat{\boldsymbol{\alpha}}_{m}(b)\right)\right),
$$

where the first term is a constant, the second term is a summation of convex functions, and the third term $-f_{1}\left(\hat{\boldsymbol{\alpha}}_{m}(b)\right)$ is also convex because $f_{1}\left(\hat{\boldsymbol{\alpha}}_{m}(b)\right)$ is concave. Therefore, the objective function of (13) is a difference between two convex functions.

\section{REFERENCES}

[1] Cisco, "Cisco visual networking index: Forecast and methodology, 20132018," June 2014.

[2] H. Dhillon, R. Ganti, F. Baccelli, and J. Andrews, "Modeling and analysis of $k$-tier downlink heterogeneous cellular networks," IEEE Journal on Selected Areas in Communications, vol. 30, no. 3, pp. 550560, April 2012.

[3] J. Andrews, S. Buzzi, W. Choi, S. Hanly, A. Lozano, A. Soong, and J. Zhang, "What will 5G be?" IEEE Journal on Selected Areas in Communications, vol. 32, no. 6, pp. 1065-1082, June 2014.

[4] J. Andrews, "Seven ways that HetNets are a cellular paradigm shift," IEEE Communications Magazine, vol. 51, no. 3, pp. 136-144, March 2013.

[5] A. Tall, Z. Altman, and E. Altman, "Self organizing strategies for enhanced ICIC (eICIC)," CoRR, vol. abs/1401.2369, 2014. [Online]. Available: http://arxiv.org/abs/1401.2369

\footnotetext{
${ }^{7}$ The domain of $f_{2, v}\left(\hat{\boldsymbol{\alpha}}_{m}(b)\right)$ is clearly convex.
} 
[6] S. Deb, P. Monogioudis, J. Miernik, and J. Seymour, "Algorithms for enhanced inter-cell interference coordination (eICIC) in LTE HetNets," IEEE/ACM Transactions on Networking, vol. 22, no. 1, pp. 137-150, February 2014.

[7] J. Pang, J. Wang, D. Wang, G. Shen, Q. Jiang, and J. Liu, "Optimized time-domain resource partitioning for enhanced inter-cell interference coordination in heterogeneous networks," in Proc. IEEE Wireless Communications and Networking Conference (WCNC), April 2012, pp. $1613-1617$.

[8] R. Thakur, A. Sengupta, and C. Siva Ram Murthy, "Improving capacity and energy efficiency of femtocell based cellular network through cell biasing," in Proc. 11th International Symposium on Modeling Optimization in Mobile, Ad Hoc Wireless Networks (WiOpt), May 2013, pp. 436443.

[9] A. Bedekar and R. Agrawal, "Optimal muting and load balancing for eICIC," in Proc. 11th International Symposium on Modeling Optimization in Mobile, Ad Hoc Wireless Networks (WiOpt), May 2013, pp. 280 287.

[10] M. Simsek, M. Bennis, and I. Güvenç, "Enhanced intercell interference coordination in HetNets: Single vs. multiflow approach," in Proc. IEEE Globecom Workshops (GC Wkshps), Dec 2013, pp. 725-729.

[11] —, "Learning based frequency- and time-domain inter-cell interference coordination in HetNets," CoRR, vol. abs/1411.5548, 2014. [Online]. Available: http://arxiv.org/abs/1411.5548

[12] A. Liu, V. K. N. Lau, L. Ruan, J. Chen, and D. Xiao, "Hierarchical radio resource optimization for heterogeneous networks with enhanced intercell interference coordination (eICIC)," IEEE Transactions on Signal Processing, vol. 62, no. 7, pp. 1684-1693, April 2014.

[13] N. Trabelsi, C. S. Chen, R. E. Azouzi, L. Roullet, and E. Altman, "User association and resource allocation optimization in LTE cellular networks," IEEE Transactions on Network and Service Management, vol. 14, no. 2, pp. 429-440, June 2017.

[14] Y. Liu, C. S. Chen, and C. W. Sung, "Distributed enhanced inter-cell interference coordination (eICIC) in LTE-Advanced HetNets: A potential game approach," in Proc. IEEE 81st Vehicular Technology Conference (VTC Spring), May 2015, pp. 1-5.

[15] — "Joint optimization on inter-cell interference management and user attachment in LTE-A HetNets," in Proc. 13th International Symposium on Modeling and Optimization in Mobile, Ad Hoc, and Wireless Networks (WiOpt), May 2015, pp. 62-69.

[16] A. Merwaday, S. Mukherjee, and I. Güvenç, "Capacity analysis of LTEAdvanced HetNets with reduced power subframes and range expansion," EURASIP Journal on Wireless Communications and Networking, vol. 2014, no. 1, pp. 1-19, 2014.

[17] J. Wang and T. Ng, Advances in $3 G$ Enhanced Technologies for Wireless Communications, ser. Artech House mobile communications series. Artech House, 2002.

[18] C. W. Sung, K. Shum, and C. Y. Ng, "Fair resource allocation for the Gaussian broadcast channel with ISI," IEEE Transactions on Communications, vol. 57, no. 5, pp. 1381-1389, May 2009.

[19] C. Singh and C. S. Chen, "Distributed downlink resource allocation in cellular networks through spatial adaptive play," in Proc. 25th International Teletraffic Congress (ITC), 2013.

[20] A. B. MacKenzie and L. A. DaSilva, Game Theory for Wireless Engineers. Morgan \& Claypool, 2006.

[21] T. Lipp and S. Boyd, "Variations and extension of the convex-concave procedure," Optimization and Engineering, pp. 1-25, 2015.

[22] S. Sesia, I. Toufik, and M. Baker, LTE - The UMTS Long Term Evolution: From Theory to Practice. Wiley, 2011.

[23] G. Caire, R. Muller, and R. Knopp, "Hard fairness versus proportional fairness in wireless communications: The single-cell case," IEEE Transactions on Information Theory, vol. 53, no. 4, pp. 1366-1385, April 2007.

[24] S. Boyd and L. Vandenberghe, Convex Optimization. New York, NY, USA: Cambridge University Press, 2004.

[25] D. Gale, The Theory of Linear Economic Models. University of Chicago Press, 1960.

[26] J. Reijnierse and J. Potters, "On finding an envy-free pareto-optimal division," Mathematical Programming, vol. 83, no. 1-3, pp. 291-311, 1998.

[27] GreenTouch, "Mobile communications WG architecture doc2: Reference scenarios," May 2013.

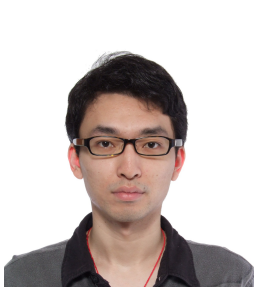

Ye Liu (M'15) received the B. Eng. in electronic and communication engineering with first class honors in 2009 and the Ph.D. in electronic engineering in 2014, both from the City University of Hong Kong, Hong Kong SAR. He is now a post-doctoral research associate with the Wolfson School of Mechanical, Electrical and Manufacturing Engineering in Loughborough University, United Kingdom. He was a visiting student of Laboratory of Information, Networking and Communication Sciences (LINCS) in Paris, France. He was also an intern in Nokia Bell Labs France. His research interests include non-orthogonal multiple access, resource allocation, and network coding.

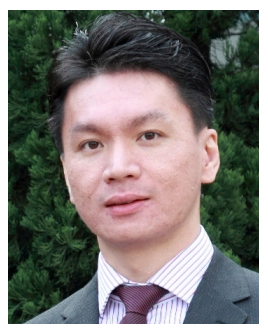

Chung Shue Chen (S'02-M'05-SM'16) received the B.Eng., M.Phil., and Ph.D. degrees in information engineering from The Chinese University of Hong Kong in 1999, 2001, and 2005, respectively.

$\mathrm{He}$ is a Member of Technical Staff (MTS) at Nokia Bell Labs. Prior to joining Bell Labs, he worked at INRIA, in the research group on Network Theory and Communications (TREC, INRIA-ENS). He was an Assistant Professor at The Chinese University of Hong Kong. He was an ERCIM Fellow at Norwegian University of Science and Technology (NTNU), Norway, and the National Center for Mathematics and Computer Science (CWI), The Netherlands. His research interests include wireless communications and networking, radio resource management, self-organizing networks, and optimization algorithms.

Dr. Chen has served as TPC in international conferences including IEEE ICC, Globecom, WCNC, VTC, CCNC, and WiOpt. He is an Editor of Transactions on Emerging Telecommunications Technologies (ETT). He also holds a position of permanent member at Laboratory of Information, Networking and Communication Sciences (LINCS), in Paris. He was the recipient of Sir Edward Youde Memorial Fellowship and ERCIM Alain Bensoussan Fellowship.

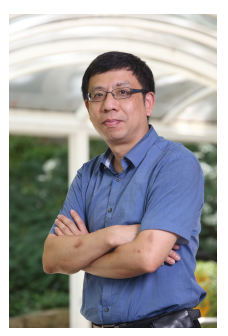

Chi Wan Sung (M'98, SM'16) received his B.Eng, M.Phil, and Ph.D. degrees in information engineering from the Chinese University of Hong Kong in 1993, 1995, and 1998, respectively. He joined the faculty at City University of Hong Kong in 2000, and is now Associate Professor with the Department of Electronic Engineering. From 2013 to 2016, he was an associate editor of the Transactions on Emerging Telecommunications Technologies (ETT). He is now on the editorial board of the ETRI Journal. He has a wide range of interests in the area of communications and networking, with emphasis on algorithm design and complexity analysis.

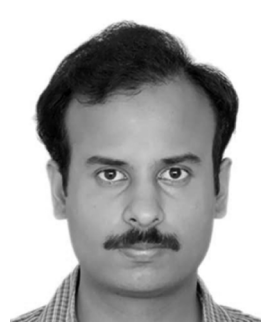

Chandramani Singh (M'14) received the M.E. and $\mathrm{Ph} . \mathrm{D}$. degrees in electrical communication engineering from the Indian Institute of Science, Bangalore, India, in 2005 and 2012, respectively.

He worked at ESQUBE Communication Solutions Pvt. Ltd., Bangalore, from 2005 to 2006. He was a Research Engineer with TREC, a joint research team between INRIA Rocquencourt and ENS de Paris, from 2012 to 2013, and a Postdoctoral Research Associate at CSL, University of Illinois at Urbana Champaign, IL, USA, from 2013 to 2014. He is now an Assistant Professor in the Department of ESE at the Indian Institute of Science, Bangalore. His interests are in the areas of communication networks, data centers and smart grids. 